\title{
Existence results of Brezis-Browder type for systems of Fredholm integral equations
}

Ravi P Agarwal ${ }^{1,2^{*}}$, Donal $\mathrm{O}^{\prime}$ Regan ${ }^{3}$ and Patricia JY Wong ${ }^{4}$

* Correspondence:

Agarwal@tamuk.edu

'Department of Mathematics, Texas

A\&M University - Kingsville,

Kingsville, TX 78363, USA

Full list of author information is

available at the end of the article

\section{Abstract}

In this article, we consider the following systems of Fredholm integral equations:

$$
\begin{array}{ll}
u_{i}(t)=h_{i}(t)+\int_{0}^{T} g_{i}(t, s) f_{i}\left(s, u_{1}(s), u_{2}(s), \ldots, u_{n}(s)\right) \mathrm{d} s, & t \in[0, T], 1 \leq i \leq n, \\
u_{i}(t)=h_{i}(t)+\int_{0}^{\infty} g_{i}(t, s) f_{i}\left(s, u_{1}(s), u_{2}(s), \ldots, u_{n}(s)\right) \mathrm{d} s, \quad t \in[0, \infty), 1 \leq i \leq n .
\end{array}
$$

Using an argument originating from Brezis and Browder [Bull. Am. Math. Soc. 81, 7378 (1975)] and a fixed point theorem, we establish the existence of solutions of the first system in $(C[0, T])^{n}$, whereas for the second system, the existence criteria are developed separately in $(C,[0, \infty))^{n}$ as well as in $(B C[0, \infty))^{n}$. For both systems, we further seek the existence of constant-sign solutions, which include positive solutions (the usual consideration) as a special case. Several examples are also included to illustrate the results obtained.

2010 Mathematics Subject Classification: 45B05; 45G15; 45M20.

Keywords: system of Fredholm integral equations, Brezis-Browder arguments, constant-sign solutions

\section{Introduction}

In this article, we shall consider the system of Fredholm integral equations:

$$
u_{i}(t)=h_{i}(t)+\int_{0}^{T} g_{i}(t, s) f_{i}\left(s, u_{1}(s), u_{2}(s), \ldots, u_{n}(s)\right) \mathrm{d} s, \quad t \in[0, T], \quad 1 \leq i \leq n
$$

where $0<T<\infty$, and also the following system on the half-line

$$
u_{i}(t)=h_{i}(t)+\int_{0}^{\infty} g_{i}(t, s) f_{i}\left(s, u_{1}(s), u_{2}(s), \ldots, u_{n}(s)\right) \mathrm{d} s, \quad t \in[0, \infty), \quad 1 \leq i \leq n .(1.2)
$$

Throughout, let $u=\left(u_{1}, u_{2}, \ldots, u_{n}\right)$. We are interested in establishing the existence of solutions $u$ of the system $(1.1)$ in $(C[0, T])^{n}=C[0, T] \times C[0, T] \times \mathbb{P} \times C[0, T](n$ times), whereas for the system (1.2), we shall seek a solution in $\left(C_{l}[0, \infty)\right)^{n}$ as well as in $(B C[0, \infty))^{n}$. Here, $B C[0, \infty)$ denotes the space of functions that are bounded and continuous on $[0, \infty)$ and $C_{l}[0, \infty)=\left\{x \in B C[0, \infty): \lim _{t \rightarrow \infty} x(t)\right.$ exists $\}$.

We shall also tackle the existence of constant-sign solutions of (1.1) and (1.2). A solution $u$ of (1.1) (or (1.2)) is said to be of constant sign if for each $1 \leq i \leq n$, we have

(c) 2011 Agarwal et al; licensee Springer. This is an Open Access article distributed under the terms of the Creative Commons Attribution License (http://creativecommons.org/licenses/by/2.0), which permits unrestricted use, distribution, and reproduction in any medium, provided the original work is properly cited. 
$\theta_{i} u_{i}(t) \geq 0$ for all $t \in[0, T]$ (or $t \in[0, \infty)$ ), where $\theta_{i} \in\{-1,1\}$ is fixed. Note that when $\theta_{i}$ $=1$ for all $1 \leq i \leq n$, a constant-sign solution reduces to a positive solution, which is the usual consideration in the literature.

In the literature, there is a vast amount of research on the existence of positive solutions of the nonlinear Fredholm integral equations:

$$
y(t)=h(t)+\int_{0}^{T} g(t, s) f(y(s)) \mathrm{d} s, \quad t \in[0, T]
$$

and

$$
y(t)=h(t)+\int_{0}^{\infty} g(t, s) f(\gamma(s)) \mathrm{d} s, \quad t \in[0, \infty) .
$$

Particular cases of (1.3) are also considered in [1-3]. The reader is referred to the monographs $[[4,5]$, and the references cited therein] for the related literature. Recently, a generalization of (1.3) and (1.4) to systems similar to (1.1) and (1.2) have been made, and the existence of single and multiple constant-sign solutions has been established for these systems in [6-10].

The technique used in these articles has relied heavily on various fixed point results such as Krasnosel'skii's fixed point theorem in a cone, Leray-Schauder alternative, Leggett-Williams' fixed point theorem, five-functional fixed point theorem, Schauder fixed point theorem, and Schauder-Tychonoff fixed point theorem. In the current study, we will make use of an argument that originates from Brezis and Browder [11]; therefore, the technique is different from those of [6-10] and the results subsequently obtained are also different. The present article also extends, improves, and complements the studies of $[5,12-23]$. Indeed, we have generalized the problems to (i) systems; (ii) more general form of nonlinearities $f_{i}, 1 \leq i \leq n$; and (iii) existence of constant-sign solutions.

The outline of the article is as follows. In Section 2, we shall state the necessary fixed point theorem and compactness criterion, which are used later. In Section 3, we tackle the existence of solutions of system $(1.1)$ in $(C[0, T])^{n}$, while Sections 4 and 5 deal with the existence of solutions of system $(1.2)$ in $\left(C_{l}[0, \infty)\right)^{n}$ and $(B C[0, \infty))^{n}$, respectively. In Section 6, we seek the existence of constant-sign solutions of (1.1) and (1.2) in $(C[0, T])^{n},\left(C_{l}[0, \infty)\right)^{n}$ and $(B C[0, \infty))^{n}$. Finally, several examples are presented in Section 7 to illustrate the results obtained.

\section{Preliminaries}

In this section, we shall state the theorems that are used later to develop the existence criteria-Theorem 2.1 [24] is Schauder's nonlinear alternative for continuous and compact maps, whereas Theorem 2.2 is the criterion of compactness on $C_{l}[0, \infty)[[16]$, p. 62].

Theorem 2.1 [24] Let $B$ be a Banach space with $E \subseteq B$ closed and convex. Assume $U$ is a relatively open subset of $E$ with $0 \in U$ and $S: \bar{U} \rightarrow$ Eis a continuous and compact map. Then either

(a) $S$ has a fixed point in $\bar{U}$, or

(b) there exist $u \in \partial U$ and $\lambda \in(0,1)$ such that $u=\lambda S u$. 
Theorem 2.2 [[16], p. 62] Let $P \subset C_{l}[0, \infty)$. Then $P$ is compact in $C_{l}[0, \infty)$ if the following hold:

(a) $P$ is bounded in $C_{l}[0, \infty)$.

(b) Any $y \in P$ is equicontinuous on any compact interval of $[0, \infty)$.

(c) $P$ is equiconvergent, i.e., given $\varepsilon>0$, there exists $T(\varepsilon)>0$ such that $|y(t)-y(\infty)|<\varepsilon$ for any $t \geq T(\varepsilon)$ and $y \in P$.

\section{Existence results for $(1.1)$ in $(C[0, T])^{n}$}

Let the Banach space $B=(C[0, T])^{n}$ be equipped with the norm:

$$
\|u\|=\max _{1 \leq i \leq n} \sup _{t \in[0, T]}\left|u_{i}(t)\right|=\max _{1 \leq i \leq n}\left|u_{i}\right|_{0}
$$

where we let $\left|u_{i}\right|_{0}=\sup _{t \in[0, T]}\left|u_{i}(t)\right|, 1 \leq i \leq n$. Throughout, for $u \in B$ and $t \in[0, T]$, we shall denote

$$
\|u(t)\|=\max _{1 \leq i \leq n}\left|u_{i}(t)\right| .
$$

Moreover, for each $1 \leq i \leq n$, let $1 \leq p_{i} \leq \infty$ be an integer and $q_{i}$ be such that $\frac{1}{p_{i}}+\frac{1}{q_{i}}=1$. For $x \in L^{p_{i}}[0, T]$, we shall define

$$
\|x\|_{p_{i}}= \begin{cases}\left(\int_{0}^{T}|x(s)|^{p_{i}} \mathrm{~d} s\right)^{\frac{1}{p_{i}}}, & 1 \leq p_{i}<\infty \\ \operatorname{ess} \sup _{s \in[0, T]}|x(s)|, & p_{i}=\infty .\end{cases}
$$

Our first existence result uses Theorem 2.1.

Theorem 3.1 For each $1 \leq i \leq n$, assume (C1)-(C4) hold where

(C1) $h_{i} \in C[0, T]$, denote $H_{i} \equiv \sup _{t \in[0, T]}\left|h_{i}(t)\right|$,

(C2) $f_{i}:[0, T] \times \mathbb{R}^{n} \rightarrow \mathbb{R}$ is a $L^{q_{i} \text { Carathéodory function: }}$

(i) the map $u \alpha f_{i}(t, u)$ is continuous for almost all $t \in[0, T]$;

(ii) the map $t \alpha f_{i}(t, u)$ is measurable for all $u \in \mathbb{R}^{n}$;

(iii) for any $r>0$, there exists $\mu_{r, i} \in L^{q_{i}}[0, T]$ such that $|u| \leq r$ implies $\left|f_{i}(t, u)\right| \leq \mu_{r, i}$

(t) for almost all $t \in[0, T]$;

(C3) $g_{i}^{t}(s)=g_{i}(t, s) \in L^{p_{i}}[0, T]$ for each $t \in[0, T]$;

(C4) the map $t \mapsto g_{i}^{t} i$ continuous from $[0, T]$ to $L^{p_{i}}[0, T]$.

In addition, suppose there is a constant $M>0$, independent of $\lambda$, with $\|u\| \neq M$ for any solution $u \in(C[0, T])^{n}$ to

$$
u_{i}(t)=\lambda\left(h_{i}(t)+\int_{0}^{T} g_{i}(t, s) f_{i}(s, u(s)) \mathrm{d} s\right), \quad t \in[0, T], \quad 1 \leq i \leq n
$$

for each $\lambda \in(0,1)$. Then, (1.1) has at least one solution in $(C[0, T])^{n}$.

Proof Let the operator $S$ be defined by

$$
S u(t)=\left(S_{1} u(t), S_{2} u(t), \ldots, S_{n} u(t)\right), \quad t \in[0, T]
$$


where

$$
S_{i} u(t)=h_{i}(t)+\int_{0}^{T} g_{i}(t, s) f_{i}(s, u(s)) \mathrm{d} s, \quad t \in[0, T], \quad 1 \leq i \leq n .
$$

Clearly, the system (1.1) is equivalent to $u=S u$, and (3.1) $\lambda$ is the same as $u=\lambda S u$.

Note that $S$ maps $(C[0, T])^{n}$ into $(C[0, T])^{n}$, i.e., $S_{i}:(C[0, T])^{n} \rightarrow C[0, T], 1 \leq i \leq n$. To see this, note that for any $u \in(C[0, T])^{n}$, there exits $r>0$ such that $\|u\|<r$. Since $f_{i}$ is a $L^{q_{i}-C a r a t h e ́ o d o r y ~ f u n c t i o n, ~ t h e r e ~ e x i s t s ~} \mu_{r, i} \in L^{q_{i}}[0, T]$ such that $\left|f_{i}(s, u)\right| \leq \mu_{r, i}(s)$ for almost all $s \in[0, T]$. Hence, for any $t_{1}, t_{2} \in[0, T]$, we find for $1 \leq i \leq n$,

$$
\left|S_{i} u\left(t_{1}\right)-S_{i} u\left(t_{2}\right)\right| \leq\left|h_{i}\left(t_{1}\right)-h_{i}\left(t_{2}\right)\right|+\left[\int_{0}^{T}\left|g_{i}^{t_{1}}(s)-g_{i}^{t_{2}}(s)\right|^{p_{i}} \mathrm{~d} s\right]^{\frac{1}{p_{i}}}\left\|\mu_{r, i}\right\|_{q_{i}} \rightarrow 0
$$

as $t_{1} \rightarrow t_{2}$, where we have used (C1) and (C3). This shows that $S:(C[0, T])^{n} \rightarrow(C[0$, $T])^{n}$.

Next, we shall prove that $S:\left(C\left[\begin{array}{ll}0, T\end{array}\right)^{n} \rightarrow(C[0, T])^{n}\right.$ is continuous. Let $u^{m}=\left(u_{1}^{m}, u_{2}^{m}, \ldots, u_{n}^{m}\right) \rightarrow u$ in $(C[0, T])^{n}$, i.e., $u_{i}^{m} \rightarrow u_{i}$ in $C[0, T], 1 \leq i \leq n$. We need to show that $S u^{m} \rightarrow S u$ in $(C[0, T])^{n}$, or equivalently $S_{i} u^{m} \rightarrow S_{i} u$ in $C[0, T], 1 \leq i \leq n$. There exists $r>0$ such that $\left\|u^{m}\right\|,\|u\|<r$. Since $f_{i}$ is a $L^{q_{i}-C a r a t h e ́ o d o r y ~ f u n c t i o n, ~}$ there exists $\mu_{r, i} \in L^{q_{i}}[0, T]$ such that $\left|f_{i}\left(s, u^{m}\right)\right|,\left|f_{i}(s, u)\right| \leq \mu_{r, i}(s)$ for almost all $s \in[0$, $T]$. Using a similar argument as in (3.4), we get for any $t_{1}, t_{2} \in[0, T]$ and $1 \leq i \leq n$ :

$$
\left|S_{i} u^{m}\left(t_{1}\right)-S_{i} u^{m}\left(t_{2}\right)\right| \rightarrow 0 \text { and }\left|S_{i} u\left(t_{1}\right)-S_{i} u\left(t_{2}\right)\right| \rightarrow 0
$$

as $t_{1} \rightarrow t_{2}$. Furthermore, $S_{i} u^{m}(t) \rightarrow S_{i} u(t)$ pointwise on [0,T], since, by the Lebesguedominated convergence theorem,

$$
\left|S_{i} u^{m}(t)-S_{i} u(t)\right| \leq\left.\sup _{t \in[0, T]}\left|g_{i}^{t}\right|\right|_{p_{i}}\left[\int_{0}^{T}\left|f_{i}\left(s, u^{m}(s)\right)-f_{i}(s, u(s))\right|^{q_{i}} \mathrm{~d} s\right]^{\frac{1}{q_{i}}} \rightarrow 0
$$

as $m \rightarrow \infty$. Combining (3.5) and (3.6) and using the fact that $[0, T]$ is compact, gives for all $t \in[0, T]$,

$$
\left|S_{i} u^{m}(t)-S_{i} u(t)\right| \leq\left|S_{i} u^{m}(t)-S_{i} u^{m}\left(t_{1}\right)\right|+\left|S_{i} u^{m}\left(t_{1}\right)-S_{i} u\left(t_{1}\right)\right|+\left|S_{i} u\left(t_{1}\right)-S_{i} u(t)\right| \rightarrow 0
$$

as $m \rightarrow \infty$. Hence, we have proved that $S:(C[0, T])^{n} \rightarrow(C[0, T])^{n}$ is continuous.

Finally, we shall show that $S:(C[0, T])^{n} \rightarrow(C[0, T])^{n}$ is completely continuous. Let $\Omega$ be a bounded set in $(C[0, T])^{n}$ with $\|u\| \leq r$ for all $u \in \Omega$. We need to show that $S_{i} \Omega$ is relatively compact for $1 \leq i \leq n$. Clearly, $S_{i} \Omega$ is uniformly bounded, since there exists $\mu_{r, i} \in L^{q_{i}}[0, T]$ such that $\left|f_{i}(s, u)\right| \leq \mu_{r, i}(s)$ for all $u \in \Omega$ and a.e. $s \in[0, T]$, and hence

$$
\left|S_{i} u\right|_{0} \leq H_{i}+\sup _{t \in[0, T]}\left\|g_{i}^{t}\right\|_{p_{i}} \cdot\left\|\mu_{r, i}\right\|_{q_{i}} \equiv K_{i}, \quad u \in \Omega
$$

Further, using a similar argument as in (3.4), we see that $S_{i} \Omega$ is equicontinuous. It follows from the Arzéla-Ascoli theorem [[5], Theorem 1.2.4] that $S_{i} \Omega$ is relatively compact.

We now apply Theorem 2.1 with $U=\left\{u \in(C[0, T])^{n}:\|u\|<M\right\}$ and $B=E=(C[0$, $T])^{n}$ to obtain the conclusion of the theorem. 
Our subsequent results will apply Theorem 3.1. To do so, we shall show that any solution $u$ of $(3.1)_{\lambda}$ is bounded above. This is achieved by bounding the integral of $\mid f_{i}(t$, $u(t)) \mid\left(\operatorname{or}\left|f_{i}(t, u(t))\right|^{\rho_{i}}\right)$ on two complementary subsets of $[0, T]$, namely $\{t \in[0, T]: \| u$ $(t) \| \leq r\}$ and $\{t \in[0, T]:\|u(t)\|>r\}$, where $\rho_{i}$ and $r$ are some constants-this technique originates from the study of Brezis and Browder [11]. In the next four theorems (Theorems 3.2-3.5), we shall apply Theorem 3.1 to the case $p_{i}=\infty$ and $q_{i}=1,1 \leq i \leq$ $n$.

Theorem 3.2. Let the following conditions be satisfied for each $1 \leq i \leq n$ : (C1)-(C4) with $p_{i}=\infty$ and $q_{i}=1,(C 5)$ and (C6) where

(C5) there exist $B_{i}>0$ such that for any $u \in(C[0, T])^{n}$,

$$
\int_{0}^{T}\left[f_{i}(t, u(t)) \int_{0}^{T} g_{i}(t, s) f_{i}(s, u(s)) \mathrm{d} s\right] \mathrm{d} t \leq B_{i}
$$

(C6) there exist $r>0$ and $\alpha_{i}>0$ with $r \alpha_{i}>H_{i}$ such that for any $u \in(C[0, T])^{n}$,

$$
u_{i}(t) f_{i}(t, u(t)) \geq r \alpha_{i}\left|f_{i}(t, u(t))\right| \text { for }\|u(t)\|>\text { rand a.e. } t \in[0, T] .
$$

Then, (1.1) has at least one solution in $(C[0, T])^{n}$.

Proof We shall employ Theorem 3.1, and so let $u=\left(u_{1}, u_{2}, 1 . \ldots, u_{n}\right) \in(C[0, T])^{n}$ be any solution of $(3.1)_{\lambda}$ where $\lambda \in(0,1)$.

Define

$$
I=\{t \in[0, T]:\|u(t)\| \leq r\} \quad \text { and } \quad J=\{t \in[0, T]:\|u(t)\|>r\} .
$$

Clearly, $[0, T]=I \cup J$, and hence $\int_{0}^{T}=\int_{I}+\int_{J}$.

Let $1 \leq i \leq n$. If $t \in I$, then by (C2), there exists $\mu_{r, i} \in L^{1}[0, T]$ such that $\left|f_{i}(t, u(t))\right| \leq$ $\mu_{r, i}(t)$. Thus, we get

$$
\int_{I}\left|f_{i}(t, u(t))\right| \mathrm{d} t \leq \int_{I} \mu_{r, i}(t) \mathrm{d} t \leq \int_{0}^{T} \mu_{r, i}(t) \mathrm{d} t=\left\|\mu_{r, i}\right\|_{1} .
$$

On the other hand, if $t \in J$, then it is clear from (C6) that $u_{i}(t) f_{i}(t, u(t)) \geq 0$ for a.e. $t$ $\in[0, T]$. It follows that

$$
\int_{J} u_{i}(t) f_{i}(t, u(t)) \mathrm{d} t \geq r \alpha_{i} \int_{J}\left|f_{i}(t, u(t))\right| \mathrm{d} t .
$$

We now multiply $(3.1)_{\lambda}$ by $f_{i}(t, u(t))$, then integrate from 0 to $T$ to get

$$
\int_{0}^{T} u_{i}(t) f_{i}(t, u(t)) \mathrm{d} t=\lambda \int_{0}^{T} h_{i}(t) f_{i}(t, u(t)) \mathrm{d} t+\lambda \int_{0}^{T}\left[f_{i}(t, u(t)) \int_{0}^{T} g_{i}(t, s) f_{i}(s, u(s)) \mathrm{d} s\right] \mathrm{d} t .
$$

Using (C5) in (3.12) yields

$$
\int_{0}^{T} u_{i}(t) f_{i}(t, u(t)) \mathrm{d} t \leq H_{i} \int_{0}^{T}\left|f_{i}(t, u(t))\right| \mathrm{d} t+B_{i}
$$


Splitting the integrals in (3.13) and applying (3.11), we get

$$
\int_{I} u_{i}(t) f_{i}(t, u(t)) \mathrm{d} t+r \alpha_{i} \int_{J}\left|f_{i}(t, u(t))\right| \mathrm{d} t \leq H_{i} \int_{I}\left|f_{i}(t, u(t))\right| \mathrm{d} t+H_{i} \int_{J}\left|f_{i}(t, u(t))\right| \mathrm{d} t+B_{i}
$$

or

$$
\begin{aligned}
\left(r \alpha_{i}-H_{i}\right) \int_{J}\left|f_{i}(t, u(t))\right| \mathrm{d} t & \leq H_{i} \int_{I}\left|f_{i}(t, u(t))\right| \mathrm{d} t+\int_{I}\left|u_{i}(t) f_{i}(t, u(t))\right| \mathrm{d} t+B_{i} \\
& \leq\left(H_{i}+r\right)\left\|\mu_{r, i}\right\|_{1}+B_{i}
\end{aligned}
$$

where we have used (3.10) in the last inequality. It follows that

$$
\int_{J}\left|f_{i}(t, u(t))\right| \mathrm{d} t \leq \frac{\left(H_{i}+r\right)\left\|\mu_{r, i}\right\|_{1}+B_{i}}{r \alpha_{i}-H_{i}} \equiv k_{i} .
$$

Finally, it is clear from $(3.1)_{\lambda}$ that for $t \in[0, T]$ and $1 \leq i \leq n$,

$$
\begin{aligned}
\left|u_{i}(t)\right| & \leq H_{i}+\int_{0}^{T}\left|g_{i}(t, s) f_{i}(s, u(s))\right| \mathrm{d} s \\
& =H_{i}+\left(\int_{I}+\int_{J}\right)\left|g_{i}(t, s) f_{i}(s, u(s))\right| \mathrm{d} s \\
& \leq H_{i}+\left(\sup _{t \in[0, T]}\left\|g_{i}^{t}\right\|_{\infty}\right)\left(\left\|\mu_{r, i}\right\|_{1}+k_{i}\right) \equiv l_{i}
\end{aligned}
$$

where we have applied (3.10) and (3.14) in the last inequality. Thus, $\left|u_{i}\right|_{0} \leq l_{i}$ for $1 \leq$ $i \leq n$ and $\|u\| \leq \max _{1 \leq i \leq n} l_{i} \equiv L$. It follows from Theorem 3.1 (with $M=L+1$ ) that (1.1) has a solution $u^{*} \in(C[0, T])^{n}$.

Theorem 3.3 Let the following conditions be satisfied for each $1 \leq i \leq n$ : (C1)-(C4) with $p_{i}=\infty$ and $q_{i}=1,(C 7)$ and (C8) where

(C7) there exist constants $a_{i} \geq 0$ and $b_{i}$ such that for any $u \in(C[0, T])^{n}$,

$$
\int_{0}^{T}\left[f_{i}(t, u(t)) \int_{0}^{T} g_{i}(t, s) f_{i}(s, u(s)) \mathrm{d} s\right] \mathrm{d} t \leq a_{i} \int_{0}^{T}\left|f_{i}(t, u(t))\right| \mathrm{d} t+b_{i},
$$

(C8) there exist $r>0$ and $\alpha_{i}>0$ with $r \alpha_{i}>H_{i}+a_{i}$ such that for any $u \in(C[0, T])^{n}$,

$$
u_{i}(t) f_{i}(t, u(t)) \geq r \alpha_{i}\left|f_{i}(t, u(t))\right| \text { for }\|u(t)\|>r \text { and a.e. } t \in[0, T] .
$$

Then, (1.1) has at least one solution in $(C[0, T])^{n}$.

Proof The proof follows that of Theorem 3.2 until (3.12). Let $1 \leq i \leq n$. We use (C7) in (3.12) to get

$$
\begin{aligned}
\int_{0}^{T} u_{i}(t) f_{i}(t, u(t)) \mathrm{d} t & \leq \int_{0}^{T}\left|h_{i}(t) f_{i}(t, u(t))\right| \mathrm{d} t+\lambda \int_{0}^{T}\left[f_{i}(t, u(t)) \int_{0}^{T} g_{i}(t, s) f_{i}(s, u(s)) \mathrm{d} s\right] \mathrm{d} t \\
& \leq\left(H_{i}+a_{i}\right) \int_{0}^{T}\left|f_{i}(t, u(t))\right| \mathrm{d} t+\left|b_{i}\right| .
\end{aligned}
$$


Splitting the integrals in (3.16) and applying (3.11) gives

$$
\begin{aligned}
\left(r \alpha_{i}-H_{i}-a_{i}\right) \int_{J}\left|f_{i}(t, u(t))\right| \mathrm{d} t & \leq\left(H_{i}+a_{i}\right) \int_{I}\left|f_{i}(t, u(t))\right| \mathrm{d} t+\int_{I}\left|u_{i}(t) f_{i}(t, u(t))\right| \mathrm{d} t+\left|b_{i}\right| \\
& \leq\left(H_{i}+a_{i}+r\right)|| \mu_{r, i} \|_{1}+\left|b_{i}\right|
\end{aligned}
$$

where we have also used (3.10) in the last inequality. It follows that

$$
\int_{J}\left|f_{i}(t, u(t))\right| \mathrm{d} t \leq \frac{\left(H_{i}+a_{i}+r\right)|| \mu_{r, i}\left|\|_{1}+\right| b_{i} \mid}{r \alpha_{i}-H_{i}-a_{i}} \equiv k_{i} .
$$

The rest of the proof follows that of Theorem 3.2.

Theorem 3.4 Let the following conditions be satisfied for each $1 \leq i \leq n$ : (C1)-(C4) with $p_{i}=\infty$ and $q_{i}=1$, (C9) and (C10) where

(C9) there exist constants $a_{i} \geq 0,0<\tau_{i} \leq 1$ and $b_{i}$ such that for any $u \in(C[0, T])^{n}$,

$$
\int_{0}^{T}\left[f_{i}(t, u(t)) \int_{0}^{T} g_{i}(t, s) f_{i}(s, u(s)) \mathrm{d} s\right] \mathrm{d} t \leq a_{i}\left[\int_{0}^{T}\left|f_{i}(t, u(t))\right| \mathrm{d} t\right]^{\tau_{i}}+b_{i},
$$

(C10) there exist $r>0$ and $\beta_{i}>0$ such that for any $u \in(C[0, T])^{n}$,

$$
u_{i}(t) f_{i}(t, u(t)) \geq \beta_{i}\|u(t)\| \cdot\left|f_{i}(t, u(t))\right| \text { for }\|u(t)\|>r \text { and a.e. } t \in[0, T] .
$$

Then, (1.1) has at least one solution in $(C[0, T])^{n}$.

Proof Let $u=\left(u_{1}, u_{2}, \ldots, u_{n}\right) \in(C[0, T])^{n}$ be any solution of $(3.1)_{\lambda}$ where $\lambda \in(0,1)$. Define

$$
\begin{gathered}
r_{0}=\max \left\{r, \max _{1 \leq i \leq n} \frac{H_{i}+a_{i} 2^{\tau_{i}}+1}{\beta_{i}}\right\}, \\
I_{0}=\left\{t \in[0, T]:\|u(t)\| \leq r_{0}\right\} \text { and } J_{0}=\left\{t \in[0, T]:\|u(t)\|>r_{0}\right\} .
\end{gathered}
$$

Clearly, $[0, T]=I_{0} \cup J_{0}$ and hence $\int_{0}^{T}=\int_{I_{0}}+\int_{J_{0}}$.

Let $1 \leq i \leq n$. If $t \in I_{0}$, then by (C2) there exists $\mu_{r_{0}, i} \in L^{1}[0, T]$ such that $\left|f_{i}(t, u(t))\right| \leq \mu_{r_{0}, i}(t)$ and

$$
\int_{I_{0}}\left|f_{i}(t, u(t))\right| \mathrm{d} t \leq \int_{I_{0}} \mu_{r_{0}, i}(t) \mathrm{d} t \leq \int_{0}^{T} \mu_{r_{0}, i}(t) \mathrm{d} t=\left\|\mu_{r_{0}, i}\right\|_{1} .
$$

Further, if $t \in J_{0}$, then by (C10) we have

$$
\int_{J_{0}} u_{i}(t) f_{i}(t, u(t)) \mathrm{d} t \geq \beta_{i} \int_{J_{0}}\|u(t)\| \cdot\left|f_{i}(t, u(t))\right| \mathrm{d} t \geq \beta_{i} r_{0} \int_{J_{0}}\left|f_{i}(t, u(t))\right| \mathrm{d} t .
$$

Now, using (3.20) and (C9) in (3.12) gives

$$
\begin{aligned}
\beta_{i} r_{0} \int_{J_{0}}\left|f_{i}(t, u(t))\right| \mathrm{d} t \leq & \int_{I_{0}}\left|u_{i}(t) f_{i}(t, u(t))\right| \mathrm{d} t+\int_{0}^{T}\left|h_{i}(t) f_{i}(t, u(t))\right| \mathrm{d} t \\
& +a_{i}\left[\int_{0}^{T}\left|f_{i}(t, u(t))\right| \mathrm{d} t\right]_{\tau_{i}}^{T}+\left|b_{i}\right| \\
\leq & \int_{I_{0}} u_{i}(t) f_{i}(t, u(t)) \mathrm{d} t+\int_{0}^{T}\left|h_{i}(t) f_{i}(t, u(t))\right| \mathrm{d} t \\
& +a_{i} 2^{\tau_{i}}\left\{\left[\int_{I_{0}}\left|f_{i}(t, u(t))\right| \mathrm{d} t\right]^{\tau_{i}}+\left[\int_{J_{0}}\left|f_{i}(t, u(t))\right| \mathrm{d} t\right]\right\}+\left|b_{i}\right|
\end{aligned}
$$


where in the last inequality, we have made use of the inequality:

$$
(x+y)^{\alpha} \leq 2^{\alpha}\left(x^{\alpha}+y^{\alpha}\right), \quad x, y \geq 0, \quad \alpha \geq 0 .
$$

Now, noting (3.19) we find that

$$
\begin{aligned}
& \int_{I_{0}}\left|u_{i}(t) f_{i}(t, u(t))\right| \mathrm{d} t+\int_{I_{0}}\left|h_{i}(t) f_{i}(t, u(t))\right| \mathrm{d} t+a_{i} 2^{\tau_{i}}\left[\int_{I_{0}}\left|f_{i}(t, u(t))\right| \mathrm{d} t\right]^{\tau_{i}}+\left|b_{i}\right| \\
& \quad \leq\left(r_{0}+H_{i}\right)\left\|\mu_{r_{0}, i}\left|\|_{1}+a_{i} 2^{\tau_{i}}\left(|| \mu_{r_{0}, i} \|_{1}\right)^{\tau_{i}}+\right| b_{i} \mid \equiv k_{i}^{\prime}\right.
\end{aligned}
$$

Substituting (3.22) in (3.21) then yields

$$
\begin{aligned}
\beta_{i} r_{0} \int_{J_{0}}\left|f_{i}(t, u(t))\right| \mathrm{d} t & \leq \int_{J_{0}}\left|h_{i}(t) f_{i}(t, u(t))\right| \mathrm{d} t+a_{i} 2^{\tau_{i}}\left[\int_{J_{0}}\left|f_{i}(t, u(t))\right| \mathrm{d} t\right]^{\tau_{i}}+k_{i}^{\prime} \\
& \leq H_{i} \int_{J_{0}}\left|f_{i}(t, u(t))\right| \mathrm{d} t+a_{i} 2^{\tau_{i}}\left[\int_{J_{0}}\left|f_{i}(t, u(t))\right| \mathrm{d} t\right]^{\tau_{i}}+k^{\prime} .
\end{aligned}
$$

Since $\tau_{i} \leq 1$, there exists a constant $k_{i}^{\prime \prime}$ such that

$$
\left(\beta_{i} r_{0}-H_{i}-a_{i} 2^{\tau_{i}}\right) \int_{J_{0}}\left|f_{i}(t, u(t))\right| \mathrm{d} t \leq k_{i}^{\prime \prime}
$$

which leads to

$$
\int_{J_{0}}\left|f_{i}(t, u(t))\right| \mathrm{d} t \leq \frac{k^{\prime \prime}}{\beta_{i} r_{0}-H_{i}-a_{i} 2^{\tau_{i}}} \equiv k_{i} .
$$

Finally, it is clear from $(3.1)_{\lambda}$ that for $t \in[0, T]$ and $1 \leq i \leq n$,

$$
\begin{aligned}
\left|u_{i}(t)\right| & \leq H_{i}+\int_{0}^{T}\left|g_{i}(t, s) f_{i}(s, u(s))\right| \mathrm{d} s \\
& \left.=H_{i}+\left(\int_{I_{0}}^{\int}+\int\right) \mid g_{J_{0}}\right)\left|(t, s) f_{i}(s, u(s))\right| \mathrm{d} s \\
& \leq H_{i}+\left(\sup _{t \in[0, T]}\left\|g_{i}^{t}\right\|_{\infty}\right)\left(\left\|\mu_{r_{0}, i}\right\|_{1}+k_{i}\right) \equiv l_{i}
\end{aligned}
$$

where we have applied (3.19) and (3.23) in the last inequality. The conclusion now follows from Theorem 3.1.

Theorem 3.5 Let the following conditions be satisfied for each $1 \leq i \leq n$ : (C1), (C2)(C4) with $p_{i}=\infty$ and $q_{i}=1,(C 10)$, (C11) and (C12) where

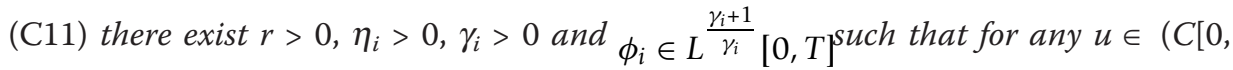
$T])^{n}$,

$$
\|u(t)\| \geq \eta_{i} \mid f_{i}\left(t,\left.u(t)\right|^{\gamma_{i}}+\phi_{i}(t) \text { for }\|u(t)\|>r \text { and a.e. } t \in[0, T]\right.
$$

(C12) there exist $a_{i} \geq 0,0<\tau_{i}<\gamma_{i}+1, b_{i}$, and $\psi_{i} \in L^{\frac{\gamma_{i}+1}{\gamma_{i}}}[0, T]^{\text {with }} \psi_{i} \geq 0$ almost everywhere on $[0, T]$, such that for any $u \in(C[0, T])^{n}$,

$$
\int_{0}^{T}\left[f_{i}(t, u(t)) \int_{0}^{T} g_{i}(t, s) f_{i}(s, u(s)) \mathrm{d} s\right] \mathrm{d} t \leq a_{i}\left[\int_{0}^{T} \psi_{i}(t)\left|f_{i}(t, u(t))\right| \mathrm{d} t\right]^{\tau_{i}}+b_{i} .
$$


Also, $\varphi_{i} \in C[0, T], h_{i} \in L^{\frac{\gamma_{i}+1}{\gamma_{i}}}[0, T]^{,} \psi_{i} \in C[0, T]$ and $\int_{0}^{T}\left|g_{i}(t, s)\right|^{\frac{\gamma_{i}+1}{\gamma_{i}}} \mathrm{~d} s \in C[0, T]$.

Then, (1.1) has at least one solution in $(C[0, T])^{n}$.

Proof Let $u=\left(u_{1}, u_{2}, \ldots, u_{n}\right) \in(C[0, T])^{n}$ be any solution of $(3.1)_{\lambda}$ where $\lambda \in(0,1)$.

Define the sets $I$ and $J$ as in (3.9). Let $1 \leq i \leq n$. Applying (C10) and (C11), we get

$$
\begin{aligned}
\int_{J} u_{i}(t) f_{i}(t, u(t)) \mathrm{d} t & \geq \beta_{i} \int_{J}\|u(t)\| \cdot\left|f_{i}(t, u(t))\right| \mathrm{d} t \\
& \geq \beta_{i} \eta_{i} \int_{J}\left|f_{i}(t, u(t))\right|^{\gamma_{i}+1} \mathrm{~d} t+\beta_{i} \int_{J} \phi_{i}(t)\left|f_{i}(t, u(t))\right| \mathrm{d} t .
\end{aligned}
$$

Using (3.25) and (C12) in (3.12), we obtain

$$
\begin{aligned}
& \beta_{i} \eta_{i} \int_{J}\left|f_{i}(t, u(t))\right|^{\gamma_{i}+1} \mathrm{~d} t \\
& \leq \int_{I}\left|u_{i}(t) f_{i}(t, u(t))\right| \mathrm{d} t+\beta_{i} \int_{J}\left|\phi_{i}(t) f_{i}(t, u(t))\right| \mathrm{d} t+\int_{0}^{T}\left|h_{i}(t) f_{i}(t, u(t))\right| \mathrm{d} t \\
& \quad+a_{i}\left[\int_{0}^{T} \psi_{i}(t)\left|f_{i}(t, u(t))\right| \mathrm{d} t\right]^{\tau_{i}}+\left|b_{i}\right| \\
& \leq \int_{I}\left|u_{i}(t) f_{i}(t, u(t))\right| \mathrm{d} t+\beta_{i} \int_{J}\left|\phi_{i}(t) f_{i}(t, u(t))\right| \mathrm{d} t+\int_{0}^{T}\left|h_{i}(t) f_{i}(t, u(t))\right| \mathrm{d} t \\
& \quad+a_{i} 2^{\tau_{i}}\left\{\left[\int_{I} \psi_{i}(t)\left|f_{i}(t, u(t))\right| \mathrm{d} t\right]^{\tau_{i}}+\left[\int_{J} \psi_{i}(t)\left|f_{i}(t, u(t))\right| \mathrm{d} t\right]^{\tau_{i}}\right\}+\left|b_{i}\right| .
\end{aligned}
$$

Now, in view of (3.10) and (C12), we have

$$
\begin{aligned}
& \int_{I}\left|u_{i}(t) f_{i}(t, u(t))\right| \mathrm{d} t+\int_{I}\left|h_{i}(t) f_{i}(t, u(t))\right| \mathrm{d} t+a_{i} 2^{\tau_{i}}\left[\int_{I} \psi_{i}(t)\left|f_{i}(t, u(t))\right| \mathrm{d} t\right]^{\tau_{i}}+\left|b_{i}\right| \\
& \leq\left.\left(r+H_{i}\right)|| \mu_{r, i}\right|_{1}+a_{i} 2^{\tau_{i}}\left[\int_{I} \psi_{i}(t) \mu_{r, i}(t) \mathrm{d} t\right]^{\tau_{i}}+\left|b_{i}\right| \equiv \bar{k}_{i} .
\end{aligned}
$$

Substituting (3.27) into (3.26) and using Hölder's inequality, we find

$$
\begin{aligned}
& \beta_{i} \eta_{i} \int_{J}\left|f_{i}(t, u(t))\right|^{\gamma_{i}+1} \mathrm{~d} t \\
& \leq \beta_{i} \int_{J}\left|\phi_{i}(t) f_{i}(t, u(t))\right| \mathrm{d} t+\int_{J}\left|h_{i}(t) f_{i}(t, u(t))\right| \mathrm{d} t+a_{i} 2^{\tau_{i}}\left[\int_{J} \psi_{i}(t)\left|f_{i}(t, u(t))\right| \mathrm{d} t\right]^{\tau_{i}}+\bar{k}_{i} \\
& \leq \beta_{i}\left[\int_{0}^{T}\left|\phi_{i}(t)\right|^{\frac{\gamma_{i}+1}{\gamma_{i}}} \mathrm{~d} t\right]^{\frac{\gamma_{i}+1}{\gamma_{i}}}\left[\int_{J}\left|f_{i}(t, u(t))\right|^{\gamma_{i}+1} \mathrm{~d} t\right]^{\frac{1}{\gamma_{i}+1}} \\
& \quad+\left[\int_{0}^{T}\left|h_{i}(t)\right|^{\frac{\gamma_{i}+1}{\gamma_{i}}} \mathrm{~d} t\right]^{\frac{\gamma_{i}}{\gamma_{i}+1}}\left[\int_{J}\left|f_{i}(t, u(t))\right|^{\gamma_{i}+1} \mathrm{~d} t\right]^{\frac{1}{\gamma_{i}+1}} \\
& \quad+a_{i} 2^{\tau_{i}}\left[\int_{0}^{T}\left|\psi_{i}(t)\right|^{\frac{\gamma_{i}+1}{\gamma_{i}}} \mathrm{~d} t\right]^{\frac{\tau_{i} \gamma_{i}}{\gamma_{i}+1}}\left[\int_{J}\left|f_{i}(t, u(t))\right|^{\gamma_{i}+1} \mathrm{~d} t\right]^{\frac{\tau_{i}}{\gamma_{i}+1}}+\bar{k}_{i} .
\end{aligned}
$$


Since $\frac{1}{\gamma_{i}+1}<1$ and $\frac{\tau_{i}}{\gamma_{i}+1}<1$, there exists a constant $k_{i}$ such that

$$
\int_{J}\left|f_{i}(t, u(t))\right|^{\gamma_{i}+1} \mathrm{~d} t \leq k_{i} .
$$

Finally, it is clear from $(3.1)_{\lambda}$ that for $t \in[0, T]$ and $1 \leq i \leq n$,

$$
\begin{aligned}
\left|u_{i}(t)\right| \leq & H_{i}+\left(\int_{I}+\int_{J}\right)\left|g_{i}(t, s) f_{i}(s, u(s))\right| \mathrm{d} s \\
\leq & H_{i}+\left(\sup _{t \in[0, T]}\left\|g_{i}^{t}\right\|_{\infty}\right)\left\|\mu_{r, i}\right\|_{1} \\
& +\left\{\sup _{t \in[0, T]}\left[\int_{0}^{T}\left|g_{i}(t, s)\right|^{\frac{\gamma_{i}+1}{\gamma_{i}}} \mathrm{~d} s\right]^{\frac{\gamma_{i}}{\gamma_{i}+1}}\right\}\left[\int_{J}\left|f_{i}(t, u(t))\right|^{\gamma_{i}+1} \mathrm{~d} t\right]^{\frac{1}{\gamma_{i}+1}} \\
\leq & l_{i}
\end{aligned}
$$

where we have used (3.28) and (C12) in the last inequality, and $l_{i}$ is some constant. The conclusion is now immediate by Theorem 3.1. $\square$

In the next six results (Theorem 3.6-3.11), we shall apply Theorem 3.1 for general $p_{i}$ and $q_{i}$.

Theorem 3.6 Let the following conditions be satisfied for each $1 \leq i \leq n$ :(C1)-(C4), (C5), (C10) and (C13) where

(C13) there exist $r>0, \eta_{i}>0, \gamma_{i}>0$ and $\phi_{i} \in L^{p_{i}}[0, T]$ such that for any $u \in(C[0, T])$ ,

$$
\|u(t)\| \geq \eta_{i} \mid f_{i}\left(t,\left.u(t)\right|^{\gamma_{i}}+\phi_{i}(t) \text { for }\|u(t)\|>r \text { and a.e. } t \in[0, T] .\right.
$$

Then, (1.1) has at least one solution in $(C[0, T])^{n}$.

Proof Let $u=\left(u_{1}, u_{2}, \ldots, u_{n}\right) \in(C[0, T])^{n}$ be any solution of $(3.1)_{\lambda}$ where $\lambda \in(0,1)$. Define the sets $I$ and $J$ as in (3.9). Let $1 \leq i \leq n$. If $t \in I$, then by (C2), there exists $\mu_{r, i} \in L^{q_{i}}[0, T]$ such that $\left|f_{i}(t, u(t))\right| \leq \mu_{r, i}(t)$. Consequently, we have

$$
\int_{I}\left|f_{i}(t, u(t))\right| \mathrm{d} t \leq \int_{I} \mu_{r, i}(t) \mathrm{d} t \leq \int_{0}^{T} \mu_{r, i}(t) \mathrm{d} t \leq T^{\frac{1}{p_{i}}} \| \mu_{r, i}||_{q_{i}} .
$$

On the other hand, using $(\mathrm{C} 10)$ and $(\mathrm{C} 13)$, we derive at (3.25).

Next, applying (C5) in (3.12) leads to (3.13). Splitting the integrals in (3.13) and using (3.25), we find that

$$
\begin{aligned}
& \beta_{i} \eta_{i} \int_{J}\left|f_{i}(t, u(t))\right|^{\gamma_{i}+1} \mathrm{~d} t \\
& \leq \beta_{i} \int_{J}\left|\phi_{i}(t) f_{i}(t, u(t))\right| \mathrm{d} t+H_{i} \int_{J}\left|f_{i}(t, u(t))\right| \mathrm{d} t+B_{i}+\int_{I}\left(\left|u_{i}(t)\right|+H_{i}\right)\left|f_{i}(t, u(t))\right| \mathrm{d} t \\
& \leq \beta_{i} \int_{J}\left|\phi_{i}(t) f_{i}(t, u(t))\right| \mathrm{d} t+H_{i} \int_{J}\left|f_{i}(t, u(t))\right| \mathrm{d} t+B_{i}+\left(r+H_{i}\right) T^{\frac{1}{p_{i}}}|| \mu_{r, i}||_{q_{i}} \\
& =\beta_{i} \int_{J}\left|\phi_{i}(t) f_{i}(t, u(t))\right| \mathrm{d} t+H_{i} \int_{J}\left|f_{i}(t, u(t))\right| \mathrm{d} t+B_{i}^{\prime}
\end{aligned}
$$

where (3.30) has been used in the last inequality and $B_{i}^{\prime} \equiv B_{i}+\left(r+H_{i}\right) T^{\frac{1}{p_{i}}}\left\|\mu_{r, i}\right\|_{q_{i}}$. 
Now, an application of Hölder's inequality gives

$$
\int_{J}\left|\phi_{i}(t) f_{i}(t, u(t))\right| \mathrm{d} t \leq\left[\int_{0}^{T}\left|\phi_{i}(t)\right|^{\frac{\gamma_{i}+1}{\gamma_{i}}} \mathrm{~d} t\right]^{\frac{\gamma_{i}}{\gamma_{i}+1}} \cdot\left[\int_{J}\left|f_{i}(t, u(t))\right|^{\gamma_{i}+1} \mathrm{~d} t\right]^{\frac{1}{\gamma_{i}+1}} .
$$

Another application of Hölder's inequality yields

$$
\int_{0}^{T}\left|\phi_{i}(t)\right|^{\frac{\gamma_{i}+1}{\gamma_{i}}} \mathrm{~d} t \leq T^{\frac{\gamma_{i} p_{i}-\gamma_{i}-1}{p_{i} \gamma_{i}}}\left[\int_{0}^{T}\left|\phi_{i}(t)\right|^{p_{i}} \mathrm{~d} t\right]^{\frac{\gamma_{i}+1}{\gamma_{i} p_{i}}} .
$$

Substituting (3.33) into (3.32) then leads to

$$
\int_{J}\left|\phi_{i}(t) f_{i}(t, u(t))\right| \mathrm{d} t \leq T^{\frac{\gamma_{i} p_{i}-\gamma_{i}-1}{p_{i}\left(\gamma_{i}+1\right)}}|| \phi_{i} \mid \|_{p_{i}}\left[\int_{J}\left|f_{i}(t, u(t))\right|^{\gamma_{i}+1} \mathrm{~d} t\right]^{\frac{1}{\gamma_{i}+1}} .
$$

Further, using Hölder's inequality again, we get

$$
\int_{J}\left|f_{i}(t, u(t))\right| \mathrm{d} t \leq T^{\frac{\gamma_{i}}{\gamma_{i}+1}}\left[\int_{J}\left|f_{i}(t, u(t))\right|^{\gamma_{i}+1} \mathrm{~d} t\right]^{\frac{1}{\gamma_{i}+1}} .
$$

Substituting (3.34) and (3.35) into (3.31), we obtain

$$
\beta_{i} \eta_{i} \int_{J}\left|f_{i}(t, u(t))\right|^{\gamma_{i}+1} \mathrm{~d} t \leq A_{i}\left[\int_{J}\left|f_{i}(t, u(t))\right|^{\gamma_{i}+1} \mathrm{~d} t\right]^{\frac{1}{\gamma_{i}+1}}+B_{i}^{\prime}
$$

where $A_{i} \equiv T^{\frac{\gamma_{i} p_{i}-\gamma_{i}-1}{p_{i}\left(\gamma_{i}+1\right)}} \beta_{i}\left\|\phi_{i}\right\|_{p_{i}}+H_{i} T^{\frac{\gamma_{i}}{\gamma_{i}+1}}$. Since $\frac{1}{\gamma_{i}+1}<1$, from (3.36), there exists a constant $k_{i}$ such that

$$
\int_{J}\left|f_{i}(t, u(t))\right|^{\gamma_{i}+1} \mathrm{~d} t \leq k_{i}
$$

Finally, it is clear from $(3.1)_{\lambda}$ that for $t \in[0, T]$ and $1 \leq i \leq n$,

$$
\begin{aligned}
\left|u_{i}(t)\right| & \leq H_{i}+\left(\int_{I}+\int_{J}\right)\left|g_{i}(t, s) f_{i}(s, u(s))\right| \mathrm{d} s \\
& \left.\leq H_{i}+\left(\sup _{t \in[0, T]}\left\|g_{i}^{t}\right\|_{p_{i}}\right)\left\|\mu_{r, i}\right\|_{q_{i}}+T^{\frac{\gamma_{i} p_{i}-\gamma_{i}-1}{p_{i}\left(\gamma_{i}+1\right)}}\left(\sup _{t \in[0, T]}\left\|g_{i}^{t}\right\| \|_{p_{i}}\right)\left[\int_{J} \mid f_{i}(s, u(s))\right)^{\gamma_{i}+1} \mathrm{~d} s\right]^{\frac{1}{\gamma_{i}+1}} \\
& \leq l_{i} \text { (a constant), }
\end{aligned}
$$

where in the second last inequality a similar argument as in (3.34) is used, and in the last inequality we have used (3.37). An application of Theorem 3.1 completes the proof. $\square$

Theorem 3.7 Let the following conditions be satisfied for each $1 \leq i \leq n$ :(C1)-(C4), (C7), (C10) and (C13). Then, (1.1) has at least one solution in $(C[0, T])^{n}$. 
Proof Let $u=\left(u_{1}, u_{2}, \ldots, u_{n}\right) \in(C[0, T])^{n}$ be any solution of $(3.1)_{\lambda}$ where $\lambda \in(0,1)$. Define the sets $I$ and $J$ as in (3.9). Let $1 \leq i \leq n$. As in the proof of Theorems 3.3 and 3.6, respectively, (C7) leads to (3.16), whereas (C10) and (C13) yield (3.25).

Splitting the integrals in (3.16) and applying (3.25), we find that

$$
\begin{aligned}
& \beta_{i} \eta_{i} \int_{J}\left|f_{i}(t, u(t))\right|^{\gamma_{i}+1} \mathrm{~d} t \\
& \leq \beta_{i} \int_{J}\left|\phi_{i}(t) f_{i}(t, u(t))\right| \mathrm{d} t+\left(H_{i}+a_{i}\right) \int_{J}\left|f_{i}(t, u(t))\right| \mathrm{d} t+\left|b_{i}\right|+\int_{I}\left(\left|u_{i}(t)\right|+H_{i}+a_{i}\right)\left|f_{i}(t, u(t))\right| \mathrm{d} t \\
& \leq \beta_{i} \int_{J}\left|\phi_{i}(t) f_{i}(t, u(t))\right| \mathrm{d} t+\left(H_{i}+a_{i}\right) \int_{J}\left|f_{i}(t, u(t))\right| \mathrm{d} t+\left|b_{i}\right|+\left(r+H_{i}+a_{i}\right) T^{\frac{1}{p_{i}}} \| \mu_{r, i}|| q_{i} \\
& =\beta_{i} \int_{J}^{J}\left|\phi_{i}(t) f_{i}(t, u(t))\right| \mathrm{d} t+\left(H_{i}+a_{i}\right) \int_{J}\left|f_{i}(t, u(t))\right| \mathrm{d} t+B^{\prime \prime} i
\end{aligned}
$$

where $B_{i}^{\prime \prime} \equiv\left|b_{i}\right|+\left(r+H_{i}+a_{i}\right) T^{\frac{1}{p_{i}}}|| \mu_{r, i} \|_{q_{i}}$. Substituting (3.34) and (3.35) into (3.39) then leads to

$$
\beta_{i} \eta_{i} \int_{J}\left|f_{i}(t, u(t))\right|^{\gamma_{i}+1} \mathrm{~d} t \leq A_{i}^{\prime}\left[\int_{J}\left|f_{i}(t, u(t))\right|^{\gamma_{i}+1} \mathrm{~d} t\right]^{\frac{1}{\gamma_{i}+1}}+B_{i}^{\prime \prime}
$$

where $A_{i}^{\prime} \equiv T^{\frac{\gamma_{i} p_{i}-\gamma_{i}-1}{p_{i}\left(\gamma_{i}+1\right)}} \beta_{i}\left\|\phi_{i}\right\|_{p_{i}}+\left(H_{i}+a_{i}\right) T^{\frac{\gamma_{i}}{\gamma_{i}+1}}$. Since $\frac{1}{\gamma_{i}+1}<1$, from (3.40), we can obtain (3.37) where $k_{i}$ is some constant. The rest of the proof proceeds as that of Theorem 3.6. $\square$

Theorem 3.8 Let the following conditions be satisfied for each $1 \leq i \leq n$ :(C1)-(C4), (C10), (C13), and (C14) where

(C14) there exist constants $a_{i} \geq 0,0<\tau_{i}<\gamma_{i}+1$ and $b_{i}$ such that for any $u \in(C[0, T])^{n}$,

$$
\int_{0}^{T}\left[f_{i}(t, u(t)) \int_{0}^{T} g_{i}(t, s) f_{i}(s, u(s)) \mathrm{d} s\right] \mathrm{d} t \leq a_{i}\left[\int_{0}^{T}\left|f_{i}(t, u(t))\right| \mathrm{d} t\right]^{\tau_{i}}+b_{i} .
$$

Then, (1.1) has at least one solution in $(C[0, T])^{n}$.

Proof Let $u=\left(u_{1}, u_{2}, \ldots, u_{n}\right) \in(C[0, T])^{n}$ be any solution of $(3.1)_{\lambda}$ where $\lambda \in(0,1)$. Define the sets $I$ and $J$ as in (3.9). Let $1 \leq i \leq n$. From the proof of Theorem 3.6, we see that $(\mathrm{C} 10)$ and $(\mathrm{C} 13)$ lead to (3.25).

Using (3.25) and (C14) in (3.12), we obtain

$$
\begin{aligned}
& \beta_{i} \eta_{i} \int_{J}\left|f_{i}(t, u(t))\right|^{\gamma_{i}+1} \mathrm{~d} t \\
& \leq \int_{I}\left|u_{i}(t) f_{i}(t, u(t))\right| \mathrm{d} t+\beta_{i} \int_{J}\left|\phi_{i}(t) f_{i}(t, u(t))\right| \mathrm{d} t+\int_{0}^{T}\left|h_{i}(t) f_{i}(t, u(t))\right| \mathrm{d} t \\
& \quad+a_{i}\left[\int_{0}^{T}\left|f_{i}(t, u(t))\right| \mathrm{d} t\right]^{\tau_{i}}+\left|b_{i}\right| \\
& \leq \int_{I}\left|u_{i}(t) f_{i}(t, u(t))\right| \mathrm{d} t+\beta_{i} \int_{J}\left|\phi_{i}(t) f_{i}(t, u(t))\right| \mathrm{d} t+\int_{0}^{T}\left|h_{i}(t) f_{i}(t, u(t))\right| \mathrm{d} t \\
& \quad+a_{i} 2^{\tau_{i}}\left\{\left[\int_{I}\left|f_{i}(t, u(t))\right| \mathrm{d} t\right]^{\tau_{i}}+\left[\int_{I}\left|f_{i}(t, u(t))\right| \mathrm{d} t\right]^{\tau_{i}}\right\}+\left|b_{i}\right| .
\end{aligned}
$$


Note that

$$
\begin{aligned}
& \int_{I}\left|u_{i}(t) f_{i}(t, u(t))\right| \mathrm{d} t+\int_{I}\left|h_{i}(t) f_{i}(t, u(t))\right| \mathrm{d} t+a_{i} 2^{\tau_{i}}\left[\int_{I}\left|f_{i}(t, u(t))\right| \mathrm{d} t\right]^{\tau_{i}}+\left|b_{i}\right| \\
& \quad \leq\left(r+H_{i}\right) \int_{I}\left|f_{i}(t, u(t))\right| \mathrm{d} t+a_{i} 2^{\tau_{i}}\left[\int_{I}\left|f_{i}(t, u(t))\right| \mathrm{d} t\right]^{\tau_{i}}+\left|b_{i}\right| \\
& \leq\left(r+H_{i}\right) T^{\frac{1}{p_{i}}}|| \mu_{r, i}||_{q_{i}}+a_{i} 2^{\tau_{i}} T^{\frac{\tau_{i}}{p_{i}}}\left(\| \mu_{r, i}||_{q_{i}}\right)^{\tau_{i}}+\left|b_{i}\right| \equiv k_{i}^{\prime}
\end{aligned}
$$

where we have used (3.30) in the last inequality. Substituting (3.42) into (3.41) and using (3.34) and (3.35) then provides

$$
\begin{aligned}
& \beta_{i} \eta_{i} \int_{J}\left|f_{i}(t, u(t))\right|^{\gamma_{i}+1} \mathrm{~d} t \\
& \leq \beta_{i} \int_{J}\left|\phi_{i}(t) f_{i}(t, u(t))\right| \mathrm{d} t+\int_{J}\left|h_{i}(t) f_{i}(t, u(t))\right| \mathrm{d} t+a_{i} 2^{\tau_{i}}\left[\int_{J}\left|f_{i}(t, u(t))\right| \mathrm{d} t\right]^{\tau_{i}}+k^{\prime} \\
& \leq \beta_{i} T^{\frac{\gamma_{i} p_{i}-\gamma_{i}-1}{p_{i}\left(\gamma_{i}+1\right)}}|| \phi_{i}||_{p_{i}}\left[\int_{J}\left|f_{i}(t, u(t))\right|^{\gamma_{i}+1} \mathrm{~d} t\right]^{\frac{1}{\gamma_{i}+1}}+H_{i} T^{\frac{\gamma_{i}}{\gamma_{i}+1}}\left[\int_{J}\left|f_{i}(t, u(t))\right|^{\gamma_{i}+1} \mathrm{~d} t\right]^{\frac{1}{\gamma_{i}+1}} \\
& \quad+a_{i} 2^{\tau_{i}} T^{\frac{\tau_{i} \gamma_{i}}{\gamma_{i}+1}}\left[\int_{J}\left|f_{i}(t, u(t))\right|^{\gamma_{i}+1} \mathrm{~d} t\right]^{\frac{\tau_{i}}{\gamma_{i}+1}}+k^{\prime} .
\end{aligned}
$$

Since $\frac{1}{\gamma_{i}+1}<1$ and $\frac{\tau_{i}}{\gamma_{i}+1}<1$, there exists a constant $k_{i}$ such that (3.37) holds. The rest of the proof is similar to that of Theorem 3.6. $\square$

Theorem 3.9 Let the following conditions be satisfied for each $1 \leq i \leq n$ : (C1)-(C4), (C10), (C13), and (C15) where

(C15) there exist constants $d_{i} \geq 0,0<\tau_{i}<\gamma_{i}+1$ and $e_{i}$ such that for any $u \in(C[0, T])^{n}$,

$$
\int_{0}^{T}\left[f_{i}(t, u(t)) \int_{0}^{T} g_{i}(t, s) f_{i}(s, u(s)) \mathrm{d} s\right] \mathrm{d} t \leq d_{i}\left[\int_{0}^{T}\left|f_{i}(t, u(t))\right|^{q_{i}} \mathrm{~d} t\right]^{\frac{\tau_{i}}{q_{i}}}+e_{i} .
$$

Then, (1.1) has at least one solution in $(C[0, T])^{n}$.

Proof Let $u=\left(u_{1}, u_{2}, \ldots, u_{n}\right) \in(C[0, T])^{n}$ be any solution of $(3.1)_{\lambda}$ where $\lambda \in(0,1)$. Define the sets $I$ and $J$ as in (3.9). Let $1 \leq i \leq n$. As before, we see that (C10) and (C13) lead to (3.25).

Using (3.25) and (C15) in (3.12), we obtain

$$
\begin{aligned}
& \beta_{i} \eta_{i} \int_{J}\left|f_{i}(t, u(t))\right|^{\gamma_{i}+1} \mathrm{~d} t \\
& \leq \beta_{i} \int_{J}\left|\phi_{i}(t) f_{i}(t, u(t))\right| \mathrm{d} t+\int_{I}\left|u_{i}(t) f_{i}(t, u(t))\right| \mathrm{d} t+\int_{0}^{T}\left|h_{i}(t) f_{i}(t, u(t))\right| \mathrm{d} t \\
& \quad+d_{i}\left[\int_{0}^{T}\left|f_{i}(t, u(t))\right|^{q_{i}} \mathrm{~d} t\right]^{\frac{\tau_{i}}{q_{i}}}+\left|e_{i}\right| \\
& \leq \beta_{i} \int_{J}\left|\phi_{i}(t) f_{i}(t, u(t))\right| \mathrm{d} t+\int_{I}\left|u_{i}(t) f_{i}(t, u(t))\right| \mathrm{d} t+H_{i} \int_{0}^{T}\left|f_{i}(t, u(t))\right| \mathrm{d} t \\
& \quad+d_{i} 2^{\frac{\tau_{i}}{q_{i}}}\left\{\left[\int_{I}\left|f_{i}(t, u(t))\right|^{q_{i}} \mathrm{~d} t\right]^{\frac{\tau_{i}}{q_{i}}}+\left[\int_{I}\left|f_{i}(t, u(t))\right|^{q_{i}} \mathrm{~d} t\right]^{\frac{\tau_{i}}{q_{i}}}\right\}+\left|e_{i}\right| .
\end{aligned}
$$


Now, it is clear that

$$
\begin{gathered}
\int_{I}\left|u_{i}(t) f_{i}(t, u(t))\right| \mathrm{d} t+H_{i} \int_{I}\left|f_{i}(t, u(t))\right| \mathrm{d} t+d_{i} 2^{\frac{\tau_{i}}{q_{i}}}\left[\int_{I}\left|f_{i}(t, u(t))\right|^{q_{i}} \mathrm{~d} t\right]^{\frac{\tau_{i}}{q_{i}}}+\left|e_{i}\right| \\
\leq\left(r+H_{i}\right) \int_{I} \mu_{r, i}(t) \mathrm{d} t+d_{i} 2^{\frac{\tau_{i}}{q_{i}}}\left[\int_{I}\left(\mu_{r, i}(t)\right)^{q_{i}} \mathrm{~d} t\right]^{\frac{\tau_{i}}{q_{i}}}+\left|e_{i}\right| \\
\leq\left(r+H_{i}\right) \int_{0}^{T} \mu_{r, i}(t) \mathrm{d} t+d_{i} 2^{\frac{\tau_{i}}{q_{i}}}\left[\int_{0}^{T}\left(\mu_{r, i}(t)\right)^{q_{i}} \mathrm{~d} t\right]^{\frac{\tau_{i}}{q_{i}}}+\left|e_{i}\right| \equiv \hat{k}_{i} .
\end{gathered}
$$

Moreover, an application of Hölder's inequality gives

$$
\int_{J}\left|f_{i}(t, u(t))\right|^{q_{i}} \mathrm{~d} t \leq T^{\frac{\gamma_{i}+1-q_{i}}{\gamma_{i}+1}}\left[\int_{J}\left|f_{i}(t, u(t))\right|^{\gamma_{i}+1} \mathrm{~d} t\right]^{\frac{q_{i}}{\gamma_{i}+1}} .
$$

Substituting (3.45) into (3.44) and using (3.34), (3.35) and (3.46) then leads to

$$
\begin{aligned}
& \beta_{i} \eta_{i} \int_{J}\left|f_{i}(t, u(t))\right|^{\gamma_{i}+1} \mathrm{~d} t \\
& \leq \beta_{i} \int_{J}\left|\phi_{i}(t) f_{i}(t, u(t))\right| \mathrm{d} t+H_{i} \int_{J}\left|f_{i}(t, u(t))\right| \mathrm{d} t+d_{i} 2^{\frac{\tau_{i}}{q_{i}}}\left[\int_{J}\left|f_{i}(t, u(t))\right|^{q_{i}} \mathrm{~d} t\right]^{\frac{\tau_{i}}{q_{i}}}+\hat{k}_{i} \\
& \leq \beta_{i} T^{\frac{\gamma_{i} p_{i}-\gamma_{i}-1}{p_{i}\left(\gamma_{i}+1\right)}}|| \phi_{i}||_{p_{i}}\left[\int_{J}\left|f_{i}(t, u(t))\right|^{\gamma_{i}+1} \mathrm{~d} t\right]^{\frac{1}{\gamma_{i}+1}}+H_{i} T^{\frac{\gamma_{i}}{\gamma_{i}+1}}\left[\int_{J}\left|f_{i}(t, u(t))\right|^{\frac{1}{\gamma_{i}+1}} \mathrm{~d} t\right]^{\frac{\tau_{i}+1}{\gamma_{i}}} \\
& \quad+d_{i} 2^{\frac{\tau_{i}}{q_{i}}} T^{\frac{\tau_{i}\left(\gamma_{i}+1-q_{i}\right)}{q_{i}\left(\gamma_{i}+1\right)}}\left[\int_{J}\left|f_{i}(t, u(t))\right|^{\gamma_{i}+1} \mathrm{~d} t\right]^{\hat{k}_{i} .}
\end{aligned}
$$

Noting $\frac{1}{\gamma_{i}+1}<1$ and $\frac{\tau_{i}}{\gamma_{i}+1}<1$, there exists a constant $k_{i}$ such that (3.37) holds. The rest of the proof follows that of Theorem 3.6.

Theorem 3.10 Let the following conditions be satisfied for each $1 \leq i \leq n$ :(C1)-(C4), (C10), (C13) and (C16) where

(C16) there exist constants $c_{i} \geq 0, d_{i} \geq 0,0<\tau_{i}<\gamma_{i}+1$ and $e_{i}$ with

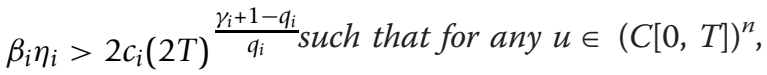

$$
\begin{aligned}
& \int_{0}^{T}\left[f_{i}(t, u(t)) \int_{0}^{T} g_{i}(t, s) f_{i}(s, u(s)) \mathrm{d} s\right] \mathrm{d} t \\
& \quad \leq c_{i}\left[\int_{0}^{T}\left|f_{i}(t, u(t))\right|^{q_{i}} \mathrm{~d} t\right]^{\frac{\gamma_{i}+1}{q_{i}}}+d_{i}\left[\int_{0}^{T}\left|f_{i}(t, u(t))\right|^{q_{i}} \mathrm{~d} t\right]^{\frac{\tau_{i}}{q_{i}}}+e_{i} .
\end{aligned}
$$

Then, (1.1) has at least one solution in $(C[0, T])^{n}$. 
Proof Let $u=\left(u_{1}, u_{2}, \ldots, u_{n}\right) \in(C[0, T])^{n}$ be any solution of $(3.1)_{\lambda}$ where $\lambda \in(0,1)$. Define the sets $I$ and $J$ as in (3.9). Let $1 \leq i \leq n$. As before, we see that (C10) and (C13) lead to (3.25).

Using (3.25) and (C16) in (3.12) gives

$$
\begin{aligned}
& \beta_{i} \eta_{i} \int_{J}\left|f_{i}(t, u(t))\right|^{\gamma_{i}+1} \mathrm{~d} t \\
& \leq \beta_{i} \int_{J}\left|\phi_{i}(t) f_{i}(t, u(t))\right| \mathrm{d} t+\int_{I}\left|u_{i}(t) f_{i}(t, u(t))\right| \mathrm{d} t+\int_{0}^{T}\left|h_{i}(t) f_{i}(t, u(t))\right| \mathrm{d} t \\
& +c_{i}\left[\int_{0}^{T}\left|f_{i}(t, u(t))\right|^{q_{i}} \mathrm{~d} t\right]^{\frac{\gamma_{i}+1}{q_{i}}}+d_{i}\left[\int_{0}^{T}\left|f_{i}(t, u(t))\right|^{q_{i}} \mathrm{~d} t\right]^{\frac{\tau_{i}}{q_{i}}}+\left|e_{i}\right| \\
& \leq \beta_{i} \int_{J}\left|\phi_{i}(t) f_{i}(t, u(t))\right| \mathrm{d} t+\int_{I}\left|u_{i}(t) f_{i}(t, u(t))\right| \mathrm{d} t+H_{i} \int_{0}^{T}\left|f_{i}(t, u(t))\right| \mathrm{d} t \\
& +c_{i} 2^{\frac{\gamma_{i}+1}{q_{i}}}\left\{\left[\int_{I}\left|f_{i}(t, u(t))\right|^{q_{i}} \mathrm{~d} t\right]^{\frac{\gamma_{i}+1}{q_{i}}}+\left[\int_{J}\left|f_{i}(t, u(t))\right|^{q_{i}} \mathrm{~d} t\right]^{\frac{\gamma_{i}+1}{q_{i}}}\right\} \\
& +d_{i} 2^{\frac{\tau_{i}}{q_{i}}}\left\{\left[\int_{I}\left|f_{i}(t, u(t))\right|^{q_{i}} \mathrm{~d} t\right]^{\frac{\tau_{i}}{q_{i}}}+\left[\int_{J}\left|f_{i}(t, u(t))\right|^{q_{i}} \mathrm{~d} t\right]^{\frac{\tau_{i}}{q_{i}}}\right\}+\left|e_{i}\right| .
\end{aligned}
$$

Now, it is clear that

$$
\begin{aligned}
& \int_{I}\left|u_{i}(t) f_{i}(t, u(t))\right| \mathrm{d} t+H_{i} \int_{I}\left|f_{i}(t, u(t))\right| \mathrm{d} t+c_{i} 2^{\frac{\gamma_{i}+1}{q_{i}}}\left[\int_{I}\left|f_{i}(t, u(t))\right|^{q_{i}} \mathrm{~d} t\right]^{\frac{\gamma_{i}+1}{q_{i}}} \\
& +d_{i} 2^{\frac{\tau_{i}}{q_{i}}}\left[\int_{I}\left|f_{i}(t, u(t))\right|^{q_{i}} \mathrm{~d} t\right]^{\frac{\tau_{i}}{q_{i}}}+\left|e_{i}\right| \\
& \leq\left(r+H_{i}\right) \int_{I} \mu_{r, i}(t) \mathrm{d} t+c_{i} 2^{\frac{\gamma_{i}+1}{q_{i}}}\left[\int_{I}\left(\mu_{r, i}(t)\right)^{q_{i}} \mathrm{~d} t\right]^{\frac{\gamma_{i}+1}{q_{i}}} \\
& +d_{i} 2^{\frac{\tau_{i}}{q_{i}}}\left[\int_{I}\left(\mu_{r, i}(t)\right)^{q_{i}} \mathrm{~d} t\right]^{\frac{\tau_{i}}{q_{i}}}+\left|e_{i}\right| \\
& \leq\left(r+H_{i}\right) \int_{0}^{T} \mu_{r, i}(t) \mathrm{d} t+c_{i} 2^{\frac{\gamma_{i}+1}{q_{i}}}\left[\int_{0}^{T}\left(\mu_{r, i}(t)\right)^{q_{i}} \mathrm{~d} t\right]^{\frac{\gamma_{i}+1}{q_{i}}} \\
& +d_{i} 2^{\frac{\tau_{i}}{q_{i}}}\left[\int_{0}^{T}\left(\mu_{r, i}(t)\right)^{q_{i}} \mathrm{~d} t\right]^{\frac{\tau_{i}}{q_{i}}}+\left|e_{i}\right| \equiv k_{i}^{\prime} .
\end{aligned}
$$


Substituting (3.49) into (3.48) and then using (3.34), (3.35) and (3.46) leads to

$$
\begin{aligned}
& \beta_{i} \eta_{i} \int_{J}\left|f_{i}(t, u(t))\right|^{\gamma_{i}+1} \mathrm{~d} t \\
& \left.\leq \beta_{i} T^{\frac{\gamma_{i} p_{i}-\gamma_{i}-1}{\left.\gamma_{i}+1\right)}} \| \phi_{i}||_{i}\left[\int_{J} \mid f_{i}(t, u(t))\right)^{\gamma_{i}+1} \mathrm{~d} t\right]^{\frac{1}{\gamma_{i}+1}}+H_{i} T^{\frac{\gamma_{i}}{\gamma_{i}+1}}\left[\int_{J}\left|f_{i}(t, u(t))\right|^{\gamma_{i+1}+1} \mathrm{~d} t\right]^{\frac{1}{\gamma_{i}+1}} \\
& +c_{i} 2^{\frac{\gamma_{i}+1}{q_{i}}} T^{\frac{\gamma_{i}+1-q_{i}}{q_{i}}} \int_{J}\left|f_{i}(t, u(t))\right|^{\gamma_{i}+1} \mathrm{~d} t \\
& +d_{i} 2^{\frac{\tau_{i}}{q_{i}}} T^{\frac{\tau_{i}\left(\gamma_{i}+1-q_{i}\right)}{q_{i}\left(\gamma_{i}+1\right)}}\left[\int_{J}\left(f_{i}(t, u(t))\right)^{\gamma_{i}+1} \mathrm{~d} t\right]^{\frac{\tau_{i}}{\gamma_{i}+1}}+k_{i}^{\prime} .
\end{aligned}
$$

Noting $\frac{1}{\gamma_{i}+1}<1, \frac{\tau_{i}}{\gamma_{i}+1}<1$ as well as $\beta_{i} \eta_{i}>2 c_{i}(2 T)^{\frac{\gamma_{i}+1-q_{i}}{q_{i}}}$, from (3.50) there exists a constant $k_{i}$ such that (3.37) holds. The rest of the proof proceeds as that of Theorem 3.6. $\square$

Theorem 3.11 Let the following conditions be satisfied for each $1 \leq i \leq n$ :(C1)-(C4), (C10), (C13) and (C17) where

(C17) there exist $a_{i} \geq 0,0<\tau_{i}<\gamma_{i}+1, b_{i}$, and $\psi_{i} \in L^{p_{i}}[0, T]$ with $\psi_{i} \geq 0$ almost everywhere on $[0, T]$, such that for any $u \in(C[0, T])^{n}$,

$$
\int_{0}^{T}\left[f_{i}(t, u(t)) \int_{0}^{T} g_{i}(t, s) f_{i}(s, u(s)) \mathrm{d} s\right] \mathrm{d} t \leq a_{i}\left[\int_{0}^{T} \psi_{i}(t)\left|f_{i}(t, u(t))\right| \mathrm{d} t\right]^{\tau_{i}}+b_{i} .
$$

Then, (1.1) has at least one solution in $(C[0, T])^{n}$.

Proof Let $u=\left(u_{1}, u_{2}, \ldots, u_{n}\right) \in(C[0, T])^{n}$ be any solution of $(3.1)_{\lambda}$ where $\lambda \in(0,1)$. Define the sets $I$ and $J$ as in (3.9). Let $1 \leq i \leq n$. Once again, conditions (C10) and (C13) give rise to (3.25).

Similar to the proof of Theorem 3.5, we apply (3.25) and (C17) in (3.12) to get (3.26). Next, using (3.30) and Hölder's inequality, we find that

$$
\begin{aligned}
& \int_{I}\left|u_{i}(t) f_{i}(t, u(t))\right| \mathrm{d} t+\int_{I}\left|h_{i}(t) f_{i}(t, u(t))\right| \mathrm{d} t+a_{i} 2^{\tau_{i}}\left[\int_{I} \psi_{i}(t)\left|f_{i}(t, u(t))\right| \mathrm{d} t\right]^{\tau_{i}}+\left|b_{i}\right| \\
& \leq\left(r+H_{i}\right) T^{\frac{1}{p_{i}}}\left\|\mu_{r, i}\left|\|_{q_{i}}+a_{i} 2^{\tau_{i}}\left[\int_{I} \psi_{i}(t) \mu_{r, i}(t) \mathrm{d} t\right]^{\tau_{i}}+\right| b_{i} \mid\right. \\
& \leq\left(r+H_{i}\right) T^{\frac{1}{p_{i}}}\left\|\mu_{r, i}\right\|_{q_{i}}+a_{i} 2^{\tau_{i}}\left(\left\|\psi_{i}\right\|\left\|_{p_{i}}|| \mu_{r, i}\right\|_{q_{i}}\right)^{\tau_{i}}+\left|b_{i}\right| \equiv k_{i}^{\prime} .
\end{aligned}
$$

Substituting (3.51) into (3.26) and applying (3.34) and (3.35), we find that

$$
\begin{aligned}
& \beta_{i} \eta_{i} \int_{J}\left|f_{i}(t, u(t))\right|^{\gamma_{i}+1} \mathrm{~d} t \\
& \leq \beta_{i} \int_{J}\left|\phi_{i}(t) f_{i}(t, u(t))\right| \mathrm{d} t+\int_{J}\left|h_{i}(t) f_{i}(t, u(t))\right| \mathrm{d} t+a_{i} 2^{\tau_{i}}\left[\int_{J} \psi_{i}(t)\left|f_{i}(t, u(t))\right| \mathrm{d} t\right]^{\tau_{i}}+k_{i}^{\prime} \\
& \left.\leq \beta_{i} T^{\frac{\chi_{i} p_{i}-\gamma_{i}-1}{p_{i}\left(\gamma_{i}+1\right)}} \| \phi_{i}||_{p_{i}}\left[\int_{J}\left|f_{i}(t, u(t))\right|^{\gamma_{i}+1} \mathrm{~d} t\right]^{\frac{1}{\gamma_{i}+1}}+H_{i} \frac{{ }^{\frac{\gamma_{i}}{\gamma_{i}+1}}}{\int}\left|f_{J}(t, u(t))\right|^{\gamma_{i}+1} \mathrm{~d} t\right]^{\frac{1}{\gamma_{i}+1}} \\
& +a_{i} 2^{\tau_{i}} T^{\frac{\tau_{i}\left(\gamma_{i} p_{i}-\gamma_{i}-1\right)}{p_{i}\left(\gamma_{i}+1\right)}}\left(\| \psi_{i}||_{p_{i}}\right)^{\tau_{i}}\left[\int_{J}\left|f_{i}(t, u(t))\right|^{\gamma_{i}+1} \mathrm{~d} t\right]^{\frac{\tau_{i}}{\gamma_{i}+1}}+k^{\prime}{ }^{\prime}
\end{aligned}
$$


Since $\frac{1}{\gamma_{i}+1}<1$ and $\frac{\tau_{i}}{\gamma_{i}+1}<1$, from (3.52), there exists a constant $k_{i}$ such that (3.37) holds. The rest of the proof proceeds as that of Theorem 3.6. $\square$

Remark 3.1 In Theorem 3.5, the conditions (C10) and (C11) can be replaced by the following, which is evident from the proof.

(C10)' There exist $r>0$ and $\beta_{i}>0$ such that for any $u \in(C[0, T])^{n}$,

$$
u_{i}(t) f_{i}(t, u(t)) \geq \beta_{i}\left|u_{i}\right|_{0} \cdot\left|f_{i}(t, u(t))\right| \text { for }\|u(t)\|>r \text { and a.e. } t \in[0, T],
$$

where we denote $\left|u_{i}\right|_{0}=\sup _{t \in[0, T]}\left|u_{i}(t)\right|$

(C11)' There exist $r>0, \eta_{i}>0, \gamma_{i}>0$ and $\phi_{i} \in L^{\frac{\gamma_{i}+1}{\gamma_{i}}}[0, T]$ such that for any $u \in(C[0$, $T])^{n}$,

$$
\left|u_{i}\right|_{0} \geq \eta_{i} \mid f_{i}\left(t,\left.u(t)\right|^{\gamma_{i}}+\phi_{i}(t) \text { for }\|u(t)\|>r \text { and a.e. } t \in[0, T] .\right.
$$

Remark 3.2 In Theorems 3.6-3.11, the conditions (C10) and (C13) can be replaced by (C10)' and (C13)' below, and the proof will be similar.

(C13)' There exist $r>0, \eta_{i}>0, \gamma_{i}>0$, and $\phi_{i} \in L^{p_{i}}[0, T]$ such that for any $u \in(C[0, T])$ ,

$$
\left|u_{i}\right|_{0} \geq \eta_{i}\left|f_{i}(t, u(t))\right|^{\gamma_{i}}+\phi_{i}(t) \text { for }\|u(t)\|>r \text { and a.e. } t \in[0, T] .
$$

\section{Existence results for $(1.2)$ in $\left(C_{l}[0, \infty)\right)^{n}$}

Let the Banach space $B=\left(C_{l}[0, \infty)\right)^{n}$ be equipped with the norm:

$$
\|u\|=\max _{1 \leq i \leq n} \sup _{t \in[0, \infty)}\left|u_{i}(t)\right|=\max _{1 \leq i \leq n}\left|u_{i}\right|_{0}
$$

where we let $\left|u_{i}\right|_{0}=\sup _{t \in[0, \infty)}\left|u_{i}(t)\right|, 1 \leq i \leq n$. Throughout, for $u \in B$ and $t \in[0$, $\infty)$, we shall denote that

$$
\|u(t)\|=\max _{1 \leq i \leq n}\left|u_{i}(t)\right| .
$$

Moreover, for each $1 \leq i \leq n$, let $1 \leq p_{i} \leq \infty$ be an integer and $q_{i}$ be such that $\frac{1}{p_{i}}+\frac{1}{q_{i}}=1$. For $x \in L^{p_{i}}[0, \infty)$, we shall define that

$$
\|x\|_{p_{i}}= \begin{cases}\left(\int_{0}^{\infty}|x(s)|^{p_{i}} \mathrm{~d} s\right)^{\frac{1}{p_{i}}}, & 1 \leq p_{i}<\infty \\ \operatorname{ess} \sup _{s \in[0, \infty)}|x(s)|, & p_{i}=\infty .\end{cases}
$$

We shall apply Theorem 2.1 to obtain the first existence result for $(1.2)$ in $\left(C_{l}[0, \infty)\right)$ $n$.

Theorem 4.1 For each $1 \leq i \leq n$, assume (D1)-(D5) hold where

(D1) $h_{i} \in C_{l}[0, \infty)$, denote $H_{i} \equiv \sup _{t \in[0, \infty)}\left|h_{i}(t)\right|$,

(D2) $f_{i}:[0, \infty) \times \mathbb{R}^{n} \rightarrow \mathbb{R}$ is a $L^{1}$-Carathéodory function, i.e.,

(i) the map $u \alpha f_{i}(t, u)$ is continuous for almost all $t \in[0, \infty)$,

(ii) the map $t \alpha f_{i}(t, u)$ is measurable for all $u \in \mathbb{R}^{n}$,

(iii) for any $r>0$, there exists $\mu_{r, i} \in L^{1}[0, \infty)$ such that $|u| \leq r$ implies $\left|f_{i}(t, u)\right| \leq \mu_{r, i}$

(t) for almost all $t \in[0, \infty)$. 
(D3) $g_{i}^{t}(s)=g_{i}(t, s) \in L^{\infty}[0, \infty)$ for each $t \in[0, \infty)$,

(D4) the map $t \mapsto g_{i}^{t}$ is continuous from $[0, \infty)$ to $L^{\infty}[0, \infty)$,

(D5) there exists $\tilde{g}_{i} \in L^{\infty}[0, \infty)$ such that $g_{i}^{t} \rightarrow \tilde{g}_{i} i n L^{\infty}[0, \infty)$ as $t \rightarrow \infty$, i.e.,

$$
\lim _{t \rightarrow \infty}|| g_{i}^{t}-\tilde{g}_{i} \|_{\infty}=\lim _{t \rightarrow \infty} e s s \sup _{s \in[0, \infty)}\left|g_{i}(t, s)-\tilde{g}_{i}(s)\right|=0 .
$$

In addition, suppose there is a constant $M>0$, independent of $\lambda$, with $\|u\| \neq M$ for any solution $u \in\left(C_{l}[0, \infty)\right)^{n}$ to

$$
u_{i}(t)=\lambda\left(h_{i}(t)+\int_{0}^{\infty} g_{i}(t, s) f_{i}(s, u(s)) \mathrm{d} s\right), \quad t \in[0, \infty), 1 \leq i \leq n
$$

for each $\lambda \in(0,1)$. Then, (1.2) has at least one solution in $\left(C_{l}[0, \infty)\right)^{n}$.

Proof To begin, let the operator $S$ be defined by

$$
S u(t)=\left(S_{1} u(t), S_{2} u(t), \ldots, S_{n} u(t)\right), \quad t \in[0, \infty)
$$

where

$$
S_{i} u(t)=h_{i}(t)+\int_{0}^{\infty} g_{i}(t, s) f_{i}(s, u(s)) \mathrm{d} s, t \in[0, \infty), \quad 1 \leq i \leq n .
$$

Clearly, the system (1.2) is equivalent to $u=S u$, and $(4.1)_{\lambda}$ is the same as $u=\lambda S u$.

First, we shall show that $S:\left(C_{l}[0, \infty)\right)^{n} \rightarrow\left(C_{l}[0, \infty)\right)^{n}$, or equivalently $S_{i}:\left(C_{l}[0, \infty)\right)^{n}$ $\rightarrow C_{l}[0, \infty), 1 \leq i \leq n$. Let $u \in\left(C_{l}[0, \infty)\right)^{n}$. Then, there exists $r>0$ such that $\|u\| \leq r$, and from (D2) there exists $\mu_{r, i} \in L^{1}[0, \infty)$ such that $\left|f_{i}(s, u)\right| \leq \mu_{r, i}(s)$ for almost all $s \in$ $[0, \infty)$. Let $t_{1}, t_{2} \in[0, \infty)$. Together with (D1) and (D4), we find that

$$
\begin{aligned}
\left|S_{i} u\left(t_{1}\right)-S_{i} u\left(t_{2}\right)\right| & \leq\left|h_{i}\left(t_{1}\right)-h_{i}\left(t_{2}\right)\right|+\int_{0}^{\infty}\left|g_{i}^{t_{1}}(s)-g_{i}^{t_{2}}(s)\right| \mu_{r, i}(s) \mathrm{d} s \\
& \leq\left|h_{i}\left(t_{1}\right)-h_{i}\left(t_{2}\right)\right|+|| g_{i}^{t_{1}}-g_{i}^{t_{2}}||_{\infty}|| \mu_{r, i} \|_{1} \rightarrow 0
\end{aligned}
$$

as $t_{1} \rightarrow t_{2}$. Hence, $S_{i} u \in C[0, \infty)$.

To see that $S_{i} u$ is bounded, we have for $t \in[0, \infty)$,

$$
\left|S_{i} u(t)\right| \leq H_{i}+\int_{0}^{\infty}\left|g_{i}(t, s)\right| \mu_{r, i}(s) \mathrm{d} s \leq H_{i}+\left\|g_{i}^{t}\right\|\left\|_{\infty}\right\| \mu_{r, i} \|_{1} .
$$

By (D5), there exists $T_{1}>0$ such that for $t>T_{1}$,

$$
\left\|g_{i}^{t}\right\|_{\infty} \leq\left\|\tilde{g}_{i}\right\|_{\infty}+1 \text {. }
$$

On the other hand, for $t \in\left[0, T_{1}\right]$, we have

$$
\left\|g_{i}^{t}\right\|_{\infty} \leq \sup _{t \in\left[0, T_{1}\right]}\left\|g_{i}^{t}\right\|_{\infty} .
$$

Hence,

$$
\sup _{t \in[0, \infty)}\left\|g_{i}^{t}\right\|_{\infty} \leq \max \left\{\sup _{t \in\left[0, T_{1}\right]}\left\|g_{i}^{t}\right\|_{\infty},\left\|\tilde{g}_{i}\right\|_{\infty}+1\right\} \equiv K_{i} .
$$


It follows from (4.5) that for $t \in[0, \infty)$,

$$
\left|S_{i} u(t)\right| \leq H_{i}+K_{i}|| \mu_{r, i} \|_{1} \equiv M_{i} .
$$

Hence, $S_{i} u$ is bounded.

It remains to check the existence of the $\operatorname{limit}_{\lim _{t \rightarrow \infty}} S_{i} u(t)$. We claim that

$$
\lim _{t \rightarrow \infty} S_{i} u(t)=h_{i}(\infty)+\int_{0}^{\infty} \tilde{g}_{i}(s) f_{i}(s, u(s)) \mathrm{d} s
$$

where $h_{i}(\infty) \equiv \lim _{t \rightarrow \infty} h_{i}(t)$. In fact, it follows from (D5) that

$$
\int_{0}^{\infty}\left|\left[g_{i}^{t}(s)-\tilde{g}_{i}(s)\right] f_{i}(s, u(s))\right| \mathrm{d} s \leq\left\|g_{i}^{t}-\tilde{g}_{i}\right\|_{\infty}\left\|\mu_{r, i}\right\|_{1} \rightarrow 0
$$

as $t \rightarrow \infty$. This implies

$$
\lim _{t \rightarrow \infty} \int_{0}^{\infty} g_{i}^{t}(s) f_{i}(s, u(s)) \mathrm{d} s=\int_{0}^{\infty} \tilde{g}_{i}(s) f_{i}(s, u(s)) \mathrm{d} s
$$

and so (4.8) is proved. We have hence shown that $S:\left(C_{l}[0, \infty)\right)^{n} \rightarrow\left(C_{l}[0, \infty)\right)^{n}$.

Next, we shall prove that $S:\left(C_{l}[0, \infty)\right)^{n} \rightarrow\left(C_{l}[0, \infty)\right)^{n}$ is continuous. Let $\left\{u^{m}\right\}$ be a sequence in $\left(C_{l}[0, \infty)\right)^{n}$ and $u^{m}=\left(u_{1}^{m}, u_{2}^{m}, \ldots, u_{n}^{m}\right) \rightarrow u$. In $\left(C_{l}[0, \infty)\right)^{n}$, i.e., $u_{i}^{m} \rightarrow u_{i}$, in $C_{l}[0, \infty), 1 \leq i \leq n$. We need to show that $S u^{m} \rightarrow S u$ in $\left(C_{l}[0, \infty)\right)^{n}$, or equivalently $S_{i} u^{m} \rightarrow S_{i} u$ in $C_{l}[0, \infty), 1 \leq i \leq n$. There exists $r>0$ such that $\left\|u^{m}\right\|,\|u\|<r$, Noting (D2), there exists $\mu_{r, i} \in L^{1}[0, \infty)$ such that $\left|f_{i}\left(s, u^{m}\right)\right|,\left|f_{i}(s, u)\right| \leq \mu_{r, i}(s)$ for almost all $s \in$ $[0, \infty)$. Denote $S_{i} u(\infty) \equiv \lim _{t \rightarrow \infty} S_{i} u(t)$ and $S_{i} u^{m}(\infty) \equiv \lim _{t \rightarrow \infty} S_{i} u^{m}(t)$. In view of (4.8), we get that

$$
\left|S_{i} u^{m}(\infty)-S_{i} u(\infty)\right| \leq \int_{0}^{\infty}\left|\tilde{g}_{i}(s)\left[f_{i}\left(s, u^{m}(s)\right)-f_{i}(s, u(s))\right]\right| \mathrm{d} s .
$$

Since

$$
\left|\tilde{g}_{i}(s)\left[f_{i}\left(s, u^{m}(s)\right)-f_{i}(s, u(s))\right]\right| \rightarrow 0 \text { as } m \rightarrow \infty \text { for almost every } s \in[0, \infty)
$$

and

$$
\left|\tilde{g}_{i}(s)\left[f_{i}\left(s, u^{m}(s)\right)-f_{i}(s, u(s))\right]\right| \leq 2 \mu_{r, i}(s)\left|\tilde{g}_{i}(s)\right| \in L^{1}[0, \infty),
$$

by the Lebesgue-dominated convergence theorem, it is clear from (4.9) that

$$
\left|S_{i} u^{m}(\infty)-S_{i} u(\infty)\right| \rightarrow 0 \text { as } m \rightarrow \infty .
$$

Further, using (4.8) again we find that

$$
\begin{aligned}
\left|S_{i} u(t)-S_{i} u(\infty)\right| & \leq\left|h_{i}(t)-h_{i}(\infty)\right|+\int_{0}^{\infty}\left|g_{i}^{t}(s)-\tilde{g}_{i}(s)\right| \mu_{r, i}(s) \mathrm{d} s \\
& \leq\left|h_{i}(t)-h_{i}(\infty)\right|+|| g_{i}^{t}-\tilde{g}_{i}||_{\infty}|| \mu_{r, i} \|_{1} \rightarrow 0
\end{aligned}
$$


as $t \rightarrow \infty$. Similarly, we also have that

$$
\left|S_{i} u^{m}(t)-S_{i} u^{m}(\infty)\right| \rightarrow 0 \text { as } t \rightarrow \infty .
$$

Combining (4.10)-(4.12), we have

$$
\left|S_{i} u^{m}(t)-S_{i} u(t)\right| \rightarrow 0 \text { as } t \rightarrow \infty \text { and } m \rightarrow \infty
$$

or equivalently, there exist $\hat{T}>0$ such that

$$
\left|S_{i} u^{m}(t)-S_{i} u(t)\right| \rightarrow 0 \text { as } m \rightarrow \infty, \text { for all } t>\hat{T} .
$$

It remains to check the convergence in $[0, \hat{T}]$. As in (4.4), we find for any $\left|S_{i} u^{m}\left(t_{1}\right)-S_{i} u^{m}\left(t_{2}\right)\right| \rightarrow 0$ and $\left|S_{i} u\left(t_{1}\right)-S_{i} u\left(t_{2}\right)\right| \rightarrow 0$,

$$
\left|S_{i} u^{m}\left(t_{1}\right)-S_{i} u^{m}\left(t_{2}\right)\right| \rightarrow 0 \text { and }\left|S_{i} u\left(t_{1}\right)-S_{i} u\left(t_{2}\right)\right| \rightarrow 0
$$

as $t_{1} \rightarrow t_{2}$. Furthermore, $S_{i} u^{m}(t) \rightarrow S_{i} u(t)$ pointwise on [0, $\left.\hat{T}\right]$, since, by the Lebesguedominated convergence theorem,

$$
\left|S_{i} u^{m}(t)-S_{i} u(t)\right| \leq \sup _{t \in[0, \hat{T}]}\left\|g_{i}^{t}\right\|_{\infty} \int_{0}^{\infty}\left|f_{i}\left(s, u^{m}(s)\right)-f_{i}(s, u(s))\right| \mathrm{d} s \rightarrow 0
$$

as $m \rightarrow \infty$. Combining (4.14) and (4.15) and the fact that $[0, \hat{T}]$ is compact yields

$$
\left|S_{i} u^{m}(t)-S_{i} u(t)\right| \rightarrow 0 \text { as } m \rightarrow \infty, \text { for all } t \in[0, \hat{T}]
$$

Coupling (4.13) and (4.16), we see that $S_{i} u^{m} \rightarrow S_{i} u$ in $C_{l}[0, \infty)$.

Finally, we shall show that $S:\left(C_{l}[0, \infty)\right)^{n} \rightarrow\left(C_{l}[0, \infty)\right)^{n}$ is completely continuous. Let $\Omega$ be a bounded set in $\left(C_{l}[0, \infty)\right)^{n}$ with $\|u\| \leq r$ for all $u \in \Omega$ We need to show that $S_{i} \Omega$ is relatively compact for $1 \leq i \leq n$. First, we see that $S_{i} \Omega$ is bounded; in fact, this follows from an earlier argument in (4.7). Next, using a similar argument as in (4.4), we see that $S_{i} \Omega$ is equicontinuous. Moreover, $S_{i} \Omega$ is equiconvergent follows as in (4.11). By Theorem 2.2, we conclude that $S_{i} \Omega$ is relatively compact. Hence, $S:\left(C_{l}[0\right.$, $\infty))^{n} \rightarrow\left(C_{l}[0, \infty)\right)^{n}$ is completely continuous.

We now apply Theorem 2.1 with $U=\left\{u \in\left(C_{l}[0, \infty)\right)^{n}:\|u\|<M\right\}$ and $B=E=\left(C_{l}[0\right.$, $\infty))^{n}$ to obtain the conclusion of the theorem. $\square$

Remark 4.1 In Theorem 4.1, the conditions (D2)-(D5) can be stated in terms of general $p_{i}$ and $q_{i}$ as follows, and the proof will be similar:

(D2)' $f_{i}:[0, \infty) \times \mathbb{R}^{n} \rightarrow \mathbb{R}$ is a $L^{q_{i-} \text { Carathéodory function, i.e., }}$

(i) the map $u \alpha f_{i}(t, u)$ is continuous for almost all $t \in[0, \infty)$,

(ii) the map $t \alpha f_{i}(t, u)$ is measurable for all $u \in \mathbb{R}^{n}$,

(iii) for any $r>0$, there exists $\mu_{r, i} \in L^{q_{i}}[0, \infty)$ such that $|u| \leq r$ implies $\left|f_{i}(t, u)\right| \leq \mu_{r, i}$

(t) for almost all $t \in[0, \infty)$,

(D3)' $g_{i}^{t}(s)=g_{i}(t, s) \in L^{p_{i}}[0, \infty)$, for each $t \in[0, \infty)$,

(D4)' the map $t \mapsto g_{i}^{t}$ is continuous from $[0, \infty)$ to $L^{p_{i}}[0, \infty)$,

(D5)' there exists $\tilde{g}_{i} \in L^{p_{i}}[0, \infty)$ such that $g_{i}^{t} \rightarrow \tilde{g}_{i}$, in $L^{p_{i}}[0, \infty)$ as $t \rightarrow \infty$, i.e.,

$$
\lim _{t \rightarrow \infty}|| g_{i}^{t}-\left.\tilde{g}_{i}\right|_{p_{i}}=\lim _{t \rightarrow \infty}\left(\int_{0}^{\infty}\left|g_{i}(t, s)-\tilde{g}_{i}(s)\right|^{p_{i}} \mathrm{~d} s\right)^{\frac{1}{p_{i}}}=0 .
$$


Our subsequent Theorems 4.2-4.5 use an argument originating from Brezis and Browder [11]. These results are parallel to Theorems 3.2-3.5 for system (1.1).

Theorem 4.2 Let the following conditions be satisfied for each $1 \leq i \leq n$ :(D1)-(D5), $(C 5)_{\infty}$ and $(C 6)_{\infty}$ where

$(\mathrm{C} 5)_{\infty}$ there exist $B_{i}>0$ such that for any $u \in\left(C_{l}[0, \infty)\right)^{n}$,

$$
\int_{0}^{\infty}\left[f_{i}(t, u(t)) \int_{0}^{\infty} g_{i}(t, s) f_{i}(s, u(s)) \mathrm{d} s\right] \mathrm{d} t \leq B_{i}
$$

(C6) $)_{\infty}$ there exist $r>0$ and $\alpha_{i}>0$ with $r \alpha_{i}>H_{i}$ such that for any $u \in\left(C_{l}[0, \infty)\right)^{n}$,

$$
u_{i}(t) f_{i}(t, u(t)) \geq r \alpha_{i}\left|f_{i}(t, u(t))\right| \text { for }\|u(t)\|>\text { r and a.e. } t \in[0, \infty) .
$$

Then, (1.2) has at least one solution in $\left(C_{l}[0, \infty)\right)^{n}$.

Proof We shall employ Theorem 4.1, so let $u=\left(u_{1}, u_{2}, \ldots, u_{n}\right) \in\left(C_{l}[0, \infty)\right)^{n}$ be any solution of $(4.1)_{\lambda}$ where $\lambda \in(0,1)$. The rest of the proof is similar to that of Theorem 3.2 with the obvious modification that $[0, T]$ be replaced by $[0, \infty)$. Also, noting (4.6) we see that the analog of (3.15) holds.

In view of the proof of Theorem 4.2, we see that the proof of subsequent Theorems 4.3-4.5 will also be similar to that of Theorems 3.3-3.5 with the appropriate modification. As such, we shall present the results and omit the proof.

Theorem 4.3 Let the following conditions be satisfied for each $1 \leq i \leq n$ :(D1)-(D5), $(\mathrm{C} 7)_{\infty}$ and $(\mathrm{C} 8)_{\infty}$ where

$(\mathrm{C} 7)_{\infty}$ there exist constants $a_{i} \geq 0$ and $b_{i}$ such that for any $u \in\left(C_{l}[0, \infty)\right)^{n}$,

$$
\int_{0}^{\infty}\left[f_{i}(t, u(t)) \int_{0}^{\infty} g_{i}(t, s) f_{i}(s, u(s)) \mathrm{d} s\right] \mathrm{d} t \leq a_{i} \int_{0}^{\infty}\left|f_{i}(t, u(t))\right| \mathrm{d} t+b_{i},
$$

$(\mathrm{C} 8)_{\infty}$ there exist $r>0$ and $\alpha_{i}>0$ with $r \alpha_{i}>H_{i}+a_{i}$ such that for any $u \in\left(C_{l}[0, \infty)\right)^{n}$,

$$
u_{i}(t) f_{i}(t, u(t)) \geq r \alpha_{i}\left|f_{i}(t, u(t))\right| \text { for }\|u(t)\|>\text { r and a.e. } t \in[0, \infty) .
$$

Then, (1.2) has at least one solution in $\left(C_{l}[0, \infty)\right)^{n}$.

Theorem 4.4 Let the following conditions be satisfied for each $1 \leq i \leq n$ :(D1)-(D5), (C9) $)_{\infty}$ and $(\mathrm{C} 10)_{\infty}$ where

(C9) $)_{\infty}$ there exist constants $a_{i} \geq 0,0<\tau_{i} \leq 1$ and $b_{i}$ such that for any $u \in\left(C_{l}[0, \infty)\right)^{n}$,

$$
\int_{0}^{\infty}\left[f_{i}(t, u(t)) \int_{0}^{\infty} g_{i}(t, s) f_{i}(s, u(s)) \mathrm{d} s\right] \mathrm{d} t \leq a_{i}\left[\int_{0}^{\infty}\left|f_{i}(t, u(t))\right| \mathrm{d} t\right]^{\tau_{i}}+b_{i},
$$

$(\mathrm{C} 10)_{\infty}$ there exist $r>0$ and $\beta_{i}>0$ such that for any $u \in\left(C_{l}[0, \infty)\right)^{n}$,

$$
u_{i}(t) f_{i}(t, u(t)) \geq \beta_{i}\|u(t)\| \cdot\left|f_{i}(t, u(t))\right| \text { for }\|u(t)\|>r \text { and a.e. } t \in[0, \infty) .
$$

Then, (1.2) has at least one solution in $\left(C_{l}[0, \infty)\right)^{n}$.

Theorem 4.5 Let the following conditions be satisfied for each $1 \leq i \leq n$ :(D1)-(D5), $(\mathrm{C} 10)_{\infty},(\mathrm{C} 11)_{\infty}$ and $(\mathrm{C} 12)_{\infty}$ where

$(\mathrm{C} 11)_{\infty}$ there exist $r>0, \eta_{i}>0, \gamma_{i}>0$ and $\phi_{i} \in L^{\frac{\gamma_{i}+1}{\gamma_{i}}}[0, \infty)^{\text {such }}$ that for any $u \in\left(C_{l}[0\right.$, $\infty))^{n}$ 


$$
\|u(t)\| \geq \eta_{i} \mid f_{i}\left(t,\left.u(t)\right|^{\gamma_{i}}+\phi_{i}(t) \text { for }\|u(t)\|>\text { r and a.e. } t \in[0, \infty),\right.
$$

$(\mathrm{C} 12)_{\infty}$ there exist $a_{i} \geq 0,0<\tau_{i}<\gamma_{i}+1, b_{i}$, and $\psi_{i} \in L^{\frac{\gamma_{i}+1}{\gamma_{i}}}[0, \infty)^{\text {with }} \psi_{i} \geq 0$ almost everywhere on $[0, \infty)$, such that for any $u \in\left(C_{l}[0, \infty)\right)^{n}$,

$$
\int_{0}^{\infty}\left[f_{i}(t, u(t)) \int_{0}^{\infty} g_{i}(t, s) f_{i}(s, u(s)) \mathrm{d} s\right] \mathrm{d} t \leq a_{i}\left[\int_{0}^{\infty} \psi_{i}(t)\left|f_{i}(t, u(t))\right| \mathrm{d} t\right]^{\tau_{i}}+b_{i} .
$$

Also, $\varphi_{i} \in B C[0, \infty), h_{i} \in L^{\frac{\gamma_{i}+1}{\gamma_{i}}}[0, \infty), \psi_{i} \in B C[0, \infty)$ and $\int_{0}^{\infty}\left|g_{i}(t, s)\right|^{\frac{\gamma_{i}+1}{\gamma_{i}}} \mathrm{~d} s \in B C[0, \infty)$.

Then, (1.2) has at least one solution in $\left(C_{l}[0, \infty)\right)^{n}$.

We also have a remark similar to Remark 3.1.

Remark 4.2 In Theorem 4.5 the conditions $(\mathrm{C} 10)_{\infty}$ and $(\mathrm{C} 11)_{\infty}$ can be replaced by the following; this is evident from the proof.

$(\mathrm{C} 10)_{\infty}^{\prime}$ There exist $r>0$ and $\beta_{i}>0$ such that for any $u \in\left(C_{l}[0, \infty)\right)^{n}$,

$$
u_{i}(t) f_{i}(t, u(t)) \geq \beta_{i}\left|u_{i}\right|_{0} \cdot\left|f_{i}(t, u(t))\right| \text { for }\|u(t)\|>r \text { and } \text { a.e. } t \in[0, \infty),
$$

where we denote $\left|u_{i}\right|_{0}=\sup _{t \in[0, \infty)}\left|u_{i}(t)\right|$.

$(\mathrm{C} 11)_{\infty}^{\prime}$ There exist $r>0, \eta_{i}>0, \gamma_{i}>0$ and $\phi_{i} \in L^{\frac{\gamma_{i}+1}{\gamma_{i}}}[0, \infty)$ such that for any $u \in$ $\left(C_{l}[0, \infty)\right)^{n}$,

$$
\left|u_{i}\right|_{0} \geq \eta_{i}\left|f_{i}(t, u(t))\right|^{\gamma_{i}}+\phi_{i}(t) \text { for }\|u(t)\|>r \text { and a.e. } t \in[0, \infty) .
$$

\section{Existence results for $(1.2)$ in $(B C[0, \infty))^{n}$}

Let the Banach space $B=(B C[0, \infty))^{n}$ be equipped with the norm:

$$
\|u\|=\max _{1 \leq i \leq n} \sup _{t \in[0, \infty)}\left|u_{i}(t)\right|=\max _{1 \leq i \leq n}\left|u_{i}\right|_{0}
$$

where we let $\left|u_{i}\right|_{0}=\sup _{t \in[0, \infty)}\left|u_{i}(t)\right|, 1<i<n$. Throughout, for $u \in B$ and $t \in[0, \infty)$ we shall denote

$$
\|u(t)\|=\max _{1 \leq i \leq n}\left|u_{i}(t)\right| .
$$

Moreover, for each $1 \leq i \leq n$, let $1 \leq p_{i} \leq \infty$ be an integer and $q_{i}$ be such that $\frac{1}{p_{i}}+\frac{1}{q_{i}}=1$. For $x \in L^{p_{i}}[0, \infty)$, we shall define $\|x\|_{p_{i}}$ as in Section 4 .

Our first result is a variation of an existence principle of Lee and O'Regan [25].

Theorem 5.1 For each $1 \leq i \leq n$, assume (D2)'-(D4)' and (D6) hold where

(D6) $h_{i} \in B C[0, \infty)$, denote $H_{i} \equiv \sup _{t \in[0, \infty)}\left|h_{i}(t)\right|$.

For each $k=1,2, \ldots$, suppose there exists $u^{k}=\left(u_{1}^{k}, u_{2}^{k}, \ldots, u_{n}^{k}\right) \in(C[0, k])^{n}$ that satisfies

$$
u_{i}^{k}(t)=h_{i}(t)+\int_{0}^{k} g_{i}(t, s) f_{i}\left(s, u_{1}^{k}(s), u_{2}^{k}(s), \ldots, u_{n}^{k}(s)\right) \mathrm{d} s, \quad t \in[0, k], \quad 1 \leq i \leq n
$$


Further, for $1 \leq i \leq n$ and $k=1,2, \ldots$, there is a bounded set $B \subseteq \mathbb{R}$ such that $u_{i}^{k}(t) \in B$ for each $t \in[0, k]$. Then, (1.2) has a solution $u^{*} \in(B C[0, \infty))^{n}$ such that for $1 \leq i \leq n, u_{i}^{*}(t) \in \bar{B}$ for all $t \in[0, \infty)$.

Proof First we shall show that

$$
\left\{\begin{array}{l}
\text { for each } 1 \leq i \leq n \text { and } \ell=1,2, \ldots, \text { the sequence }\left\{u_{i}^{k}\right\}_{k \geq \ell} \\
\text { is uniformly bounded and equicontinuous on }[0, \ell]
\end{array}\right.
$$

The uniform boundedness of $\left\{u_{i}^{k}\right\}_{k \geq \ell}$ follows immediately from the hypotheses; therefore, we only need to prove that $\left\{u_{i}^{k}\right\}_{k \geq \ell}$ is equicontinuous. Let $1 \leq i \leq n$. Since $u_{i}^{k}(t) \in B$ for each $t \in[0, k]$, there exists $\mu_{B} \in L^{q_{i}}[0, \infty)$ such that $\left|f_{i}\left(s, u^{k}(s)\right)\right| \leq \mu_{B}(s)$ for almost every $s \in[0, k]$.Fix $t, t^{\prime} \in[0, \lambda]$. Then, from (5.1) we find that

$$
\begin{aligned}
\left|u_{i}^{k}(t)-u_{i}^{k}\left(t^{\prime}\right)\right| & \leq\left|h_{i}(t)-h_{i}\left(t^{\prime}\right)\right|+\int_{0}^{k}\left|g_{i}^{t}(s)-g_{i}^{t^{\prime}}(s)\right| \cdot\left|f_{i}\left(s, u^{k}(s)\right)\right| \mathrm{d} s \\
& =\left|h_{i}(t)-h_{i}\left(t^{\prime}\right)\right|+\int_{0}^{\infty} 1_{[0, k]}\left|g_{i}^{t}(s)-g_{i}^{t^{\prime}}(s)\right| \cdot\left|f_{i}\left(s, u^{k}(s)\right)\right| \mathrm{d} s \\
& \leq\left|h_{i}(t)-h_{i}\left(t^{\prime}\right)\right|+|| g_{i}^{t}-g_{i}^{t^{\prime}}||_{p_{i}} \cdot\left\|\mu_{B}\right\|_{q_{i}} \rightarrow 0
\end{aligned}
$$

as $t \rightarrow t^{\prime}$. Therefore, $\left\{u_{i}^{k}\right\}_{k \geq \ell}$ is equicontinuous on $[0, \lambda]$.

Let $1 \leq i \leq n$. Now, (5.2) and the Arzéla-Ascoli theorem yield a subsequence $N_{1}$ of $\mathbb{N}$ $=\{1,2, \ldots\}$ and a function $z_{i}^{1} \in C[0,1]$ such that $u_{i}^{k} \rightarrow z_{i}^{1}$ uniformly on $[0,1]$ as $k \rightarrow \infty$ in $N_{1}$. Let $N_{2}^{*}=N_{1} \backslash\{1\}$. Then, (5.2) and the Arzéla-Ascoli theorem yield a subsequence $N_{2}$ of $N_{2}^{*}$ and a function $z_{i}^{2} \in C[0,2]$ such that $u_{i}^{k} \rightarrow z_{i}^{2}$ uniformly on [0,2] as $k \rightarrow \infty$ in $N_{2}$. Note that $z_{i}^{2}=z_{i}^{1}$ on $[0,1]$ since $N_{2} \subseteq N_{1}$. Continuing this process, we obtain subsequences of integers $N_{1}, N_{2}, \ldots$ with

$$
N_{1} \supseteq N_{2} \supseteq \cdots \supseteq N_{\ell} \supseteq \cdots, \quad \text { where } N_{\ell} \subseteq\{\ell, \ell+1, \ldots\},
$$

and functions $z_{i}^{\ell} \in C[0, \ell]$ such that

$$
\begin{aligned}
& u_{i}^{k} \rightarrow z_{i}^{\ell} \text { uniformly on }[0, \ell] \text { as } k \rightarrow \infty \text { in } N_{\ell,} \\
& \text { and } z_{i}^{\ell+1}=z_{i}^{\ell} \text { on }[0, \ell], \ell=1,2, \ldots
\end{aligned}
$$

Let $1 \leq i \leq n$. Define a function $u_{i}^{*}:[0, \infty] \rightarrow \mathbb{R}$ by

$$
u_{i}^{*}(t)=z_{i}^{\ell}(t), \quad t \in[0, \ell] .
$$

Clearly, $u_{i}^{*} \in C[0, \infty)$ and $u_{i}^{*}(t) \in \bar{B}$ for each $t \in[0, \lambda]$. It remains to prove that $u^{*}=\left(u_{1}^{*}, u_{2}^{*}, \ldots, u_{n}^{*}\right)$ solves (1.2). Fix $t \in[0, \infty)$. Then, choose and fix $\lambda$ such that $t \in$ $[0, \lambda]$. Take $k \geq \lambda$. Now, from (5.1) we have

$$
u_{i}^{k}(t)=h_{i}(t)+\int_{0}^{k} g_{i}(t, s) f_{i}\left(s, u_{1}^{k}(s), u_{2}^{k}(s), \ldots, u_{n}^{k}(s)\right) \mathrm{d} s, \quad t \in[0, \ell]
$$


or equivalently

$$
\begin{aligned}
& u_{i}^{k}(t)-h_{i}(t)-\int_{0}^{\ell} g_{i}(t, s) f_{i}\left(s, u_{1}^{k}(s), u_{2}^{k}(s), \ldots, u_{n}^{k}(s)\right) \mathrm{d} s \\
& \quad=\int_{l}^{k} g_{i}(t, s) f_{i}\left(s, u_{1}^{k}(s), u_{2}^{k}(s), \ldots, u_{n}^{k}(s)\right) \mathrm{d} s, \quad t \in[0, \ell] .
\end{aligned}
$$

Since $f_{i}$ is a $L^{q_{i}-C a r a t h e ́ o d o r y ~ f u n c t i o n ~ a n d ~} u_{i}^{k}(t) \in B$ for each $t \in[0, k]$, there exists $\mu_{B} \in L^{q_{i}}[0, \infty)$ such that

$$
\left|g_{i}(t, s) f_{i}\left(s, u_{1}^{k}(s), u_{2}^{k}(s), \ldots, u_{n}^{k}(s)\right)\right| \leq\left|g_{i}^{t}(s)\right| \mu_{B}(s), \quad \text { a.e. } s \in[0, k]
$$

and $\left|g_{i}^{t}\right| \mu_{B} \in L^{1}[0, \infty)$. Let $k \rightarrow \infty\left(k \in N_{\ell}\right)$ in (5.6). Since $u_{i}^{k} \rightarrow z_{i}^{\ell}$ uniformly on $[0, \ell]$, an application of Lebesgue-dominated convergence theorem gives

$$
\left|z_{i}^{\ell}(t)-h_{i}(t)-\int_{0}^{\ell} g_{i}(t, s) f_{i}\left(s, z_{1}^{\ell}(s), z_{2}^{\ell}(s), \ldots, z_{n}^{\ell}(s)\right) \mathrm{d} s\right| \leq \int_{l}^{\infty}\left|g_{i}^{t}(s)\right| \mu_{B}(s) \mathrm{d} s, \quad t \in[0, \ell]
$$

or equivalently (noting (5.5))

$$
\left|u_{i}^{*}(t)-h_{i}(t)-\int_{0}^{\ell} g_{i}(t, s) f_{i}\left(s, u_{1}^{*}(s), u_{2}^{*}(s), \ldots, u_{n}^{*}(s)\right) \mathrm{d} s\right| \leq \int_{l}^{\infty}\left|g_{i}^{t}(s)\right| \mu_{B}(s) \mathrm{d} s, \quad t \in[0, \ell] .
$$

Finally, letting $\ell \rightarrow \infty$ in (5.7) and use the fact $\left|g_{i}^{t}\right| \mu_{B} \in L^{1}[0, \infty)$ to get

$$
u_{i}^{*}(t)-h_{i}(t)-\int_{0}^{\infty} g_{i}(t, s) f_{i}\left(s, u_{1}^{*}(s), u_{2}^{*}(s), \ldots, u_{n}^{*}(s)\right) \mathrm{d} s=0, \quad t \in[0, \infty) .
$$

Hence, $u^{*}=\left(u_{1}^{*}, u_{2}^{*}, \ldots, u_{n}^{*}\right)$ is a solution of $(1.2)$.

It is noted that one of the conditions in Theorem 5.1, namely, (5.1) has a solution in $(C[0, k])^{n}$, which has already been discussed in Section 3 . As such, our subsequent Theorems 5.2-5.5 will make use of Theorem 5.1 and the technique used in Section 3. These results are parallel to Theorems 3.2-3.5 and 4.2-4.5.

Theorem 5.2 Let (D2)-(D4) and (D6) be satisfied for each $1 \leq i \leq n$. Moreover, suppose the following conditions hold for each $1 \leq i \leq n$ and each $w \in\{1,2, \ldots\}$ :

$(\mathrm{C} 5)_{w}$ there exist $B_{i}>0$ such that for any $u \in(C[0, w])^{n}$,

$$
\int_{0}^{w}\left[f_{i}(t, u(t)) \int_{0}^{w} g_{i}(t, s) f_{i}(s, u(s)) \mathrm{d} s\right] \mathrm{d} t \leq B_{i},
$$

$(\mathrm{C6})_{w}$ there exist $r>0$ and $\alpha_{i}>0$ with $r \alpha_{i}>H_{i}\left(H_{i}\right.$ as in (D6)) such that for any $u \in$ $(C[0, w])^{n}$,

$$
u_{i}(t) f_{i}(t, u(t)) \geq r \alpha_{i}\left|f_{i}(t, u(t))\right| \text { for }\|u(t)\| r \text { and a.e. } t \in[0, w] .
$$

Then, (1.2) has at least one solution in $(B C[0, \infty))^{n}$.

Proof We shall apply Theorem 5.1. To do so, for $w=1,2, \ldots$, we shall show that the system 


$$
u_{i}(t)=h_{i}(t)+\int_{0}^{w} g_{i}(t, s) f_{i}(s, u(s)) \mathrm{d} s, \quad t \in[0, w], \quad 1 \leq i \leq n
$$

has a solution in $(C[0, w])^{n}$. Obviously, (5.8) is just (1.1) with $T=w$. Let $w \in\{1,2, \ldots\}$ be fixed.

Let $u=\left(u_{1}, u_{2}, \ldots, u_{n}\right) \in(C[0, w])^{n}$ be any solution of (3.1) (with $\left.T=w\right)$ where $\lambda \in$ $(0,1)$. We shall model after the proof of Theorem 3.2 with $T=w$ and $H_{i}$ given in (D6). As in (3.9), define

$$
I=\{t \in[0, w]:\|u(t)\| \leq r\} \quad \text { and } \quad J=\{t \in[0, w]:\|u(t)\|>r\} .
$$

Let $1 \leq i \leq n$. If $t \in I$, then by (D2) there exists $\mu_{r, i} \in L^{1}[0, \infty)$ such that

$$
\int_{I}\left|f_{i}(t, u(t))\right| \mathrm{d} t \leq \int_{I} \mu_{r, i}(t) \mathrm{d} t \leq \int_{0}^{\infty} \mu_{r, i}(t) \mathrm{d} t=\left\|\mu_{r, i}\right\|_{1}
$$

[which is the analog of (3.10)]. Proceeding as in the proof of Theorem 3.2, we then obtain the analog of (3.14) as

$$
\int_{J}\left|f_{i}(t, u(t))\right| \mathrm{d} t \leq \frac{\left(H_{i}+r\right)\left\|\mu_{r, i}\right\|_{1}+B_{i}}{r \alpha_{i}-H_{i}} \equiv k_{i} \quad \text { (independent of } w \text { ). }
$$

Further, the analog of (3.15) appears as

$$
\begin{aligned}
\left|u_{i}(t)\right| & \leq \sup _{t \in[0, w]}\left|h_{i}(t)\right|+\left(\sup _{t \in[0, w]} \operatorname{ess} \sup _{s \in[0, w]}\left|g_{i}(t, s)\right|\right)\left(\left\|\mu_{r, i}\right\|_{1}+k_{i}\right) \\
& \left.\leq H_{i}+\left(\sup _{t \in[0, \infty)} \operatorname{ess} \sup _{s \in[0, \infty)}\left|g_{i}(t, s)\right|\right)\left(\left\|\mu_{r, i}\right\|_{1}+k_{i}\right) \equiv l_{i} \quad \text { (independent of } w\right), \quad t \in[0, w] .
\end{aligned}
$$

Hence, $\|u\| \leq \max _{1 \leq i \leq n} l_{i}=L$ and we conclude from Theorem 3.1 that (5.8) has a solution $u^{*}$ in $(C[0, w])^{n}$. Using similar arguments as in getting (5.9), we find $\left|u_{i}^{*}(t)\right| \leq l_{i}$ for each $t \in[0, w]$. All the conditions of Theorem 5.1 are now satisfied, it follows that (1.2) has at least one solution in $(B C[0, \infty))^{n}$.

The proof of subsequent Theorems 5.3-5.5 will model after the proof of Theorem 5.2, and will employ similar arguments as in the proof of Theorems 3.3-3.5. As such, we shall present the results and omit the proof.

Theorem 5.3 Let (D2)-(D4) and (D6) be satisfied for each $1 \leq i \leq n$. Moreover, suppose the following conditions hold for each $1 \leq i \leq n$ and each $w \in\{1,2, \ldots\}$ :

$(C 7)_{w}$ there exist constants $a_{i} \geq 0$ and $b_{i}$ such that for any $u \in(C[0, w])^{n}$,

$$
\int_{0}^{w}\left[f_{i}(t, u(t)) \int_{0}^{w} g_{i}(t, s) f_{i}(s, u(s)) \mathrm{d} s\right] \mathrm{d} t \leq a_{i} \int_{0}^{w}\left|f_{i}(t, u(t))\right| \mathrm{d} t+b_{i},
$$

(C8) $)_{w}$ there exist $r>0$ and $\alpha_{i}>0$ with $r \alpha_{i}>H_{i}+a_{i}\left(H_{i}\right.$ as in (D6)) such that for any $u \in(C[0, w])^{n}$,

$$
u_{i}(t) f_{i}(t, u(t)) \geq r \alpha_{i}\left|f_{i}(t, u(t))\right| \text { for }\|u(t)\|>r \text { and a.e. } t \in[0, w] .
$$

Then, (1.2) has at least one solution in $(B C[0, \infty))^{n}$.

Theorem 5.4 Let (D2)-(D4) and (D6) be satisfied for each $1 \leq i \leq n$. Moreover, suppose the following conditions hold for each $1 \leq i \leq n$ and each $w \in\{1,2, \ldots\}$ : 
(C9) $)_{w}$ there exist constants $a_{i} \geq 0,0<\tau_{i} \leq 1$ and $b_{i}$ such that for any $u \in(C[0, w])^{n}$,

$$
\int_{0}^{w}\left[f_{i}(t, u(t)) \int_{0}^{w} g_{i}(t, s) f_{i}(s, u(s)) \mathrm{d} s\right] \mathrm{d} t \leq a_{i}\left[\int_{0}^{w}\left|f_{i}(t, u(t))\right| \mathrm{d} t\right]^{\tau_{i}}+b_{i},
$$

$(\mathrm{C} 10)_{w}$ there exist $r>0$ and $\beta_{i}>0$ such that for any $u \in(C[0, w])^{n}$,

$$
u_{i}(t) f_{i}(t, u(t)) \geq \beta_{i}\|u(t)\| \cdot \| f_{i}(t, u(t)) \mid \text { for }\|u(t)\|>\text { r and a.e. } t \in[0, w] .
$$

Then, (1.2) has at least one solution in $(B C[0, \infty))^{n}$.

Theorem 5.5 Let (D2)-(D4) and (D6) be satisfied for each $1 \leq i \leq n$. Moreover, suppose the following conditions hold for each $1 \leq i \leq n$ and each $w \in\{1,2, \ldots\}:(C 10)_{w}$,

$(\mathrm{C} 11)_{w}$ there exist $r>0, \eta_{i}>0, \gamma_{i}>0$ and $\underset{\phi_{i} \in L}{\frac{\gamma_{i}+1}{\gamma_{i}}}[0, w]$ such that for any $u \in(C[0$, $w])^{n}$,

$$
\|u(t)\| \geq \eta_{i} \mid f_{i}\left(t,\left.u(t)\right|^{\gamma_{i}}+\phi_{i}(t) \text { for }\|u(t)\|>\text { r and a.e. } t \in[0, w],\right.
$$

$(\mathrm{C} 12)_{w}$ there exist $a_{i} \geq 0,0<\tau_{i}<\gamma_{i}+1, b_{i}$, and $\psi_{i} \in L \frac{\gamma_{i}+1}{\gamma_{i}}[0, w]$ with $\psi_{i} \geq 0$ almost everywhere on $[0, w]$, such that for any $u \in(C[0, w])^{n}$,

$$
\int_{0}^{w}\left[f_{i}(t, u(t)) \int_{0}^{w} g_{i}(t, s) f_{i}(s, u(s)) \mathrm{d} s\right] \mathrm{d} t \leq a_{i}\left[\int_{0}^{w} \psi_{i}(t)\left|f_{i}(t, u(t))\right| \mathrm{d} t\right]^{\tau_{i}}+b_{i} .
$$

Also, $\varphi_{\mathrm{i}} \in C[0, w], h_{i} \in L \frac{\gamma_{i}+1}{\gamma_{i}}[0, w] \psi_{i} \in C[0, w]$ and $\int_{0}^{w}\left|g_{i}(t, s)\right| \frac{\gamma_{i}+1}{\gamma_{i}} \mathrm{~d} s \in C[0, w]$.

Then, (1.2) has at least one solution in $(B C[0, \infty))^{n}$.

We also have a remark similar to Remark 3.1.

Remark 5.1 In Theorem 5.5 the conditions $(\mathrm{C} 10)_{w}$ and $(\mathrm{C} 11)_{w}$ can be replaced by the following, this is evident from the proof.

$(\mathrm{C} 10)_{w}^{\prime}$ There exist $r>0$ and $\beta_{i}>0$ such that for any $u \in(C[0, w])^{n}$,

$$
u_{i}(t) f_{i}(t, u(t)) \geq \beta_{i}\left|u_{i}\right|_{0} \cdot\left|f_{i}(t, u(t))\right| \text { for }\|u(t)\|>r \text { and a.e. } t \in[0, w],
$$

where we denote $\left|u_{i}\right|_{0}=\sup _{t \in[0, w]}\left|u_{i}(t)\right|$

(C11) $)_{w}^{\prime}$ There exist $r>0, \eta_{i}>0, \gamma_{i}>0$ and $\underset{\phi_{i} \in L}{\frac{\gamma_{i}+1}{\gamma_{i}}}[0, w]$ such that for any $u \in(C$ $[0, w])^{n}$,

$$
\left|u_{i}\right|_{0} \geq \eta_{i} \mid f_{i}\left(t,\left.u(t)\right|^{\gamma_{i}}+\phi_{i}(t) \text { for }\|u(t)\|>r \text { and a.e. } t \in[0, w] .\right.
$$

\section{Existence of constant-sign solutions}

In this section, we shall establish the existence of constant-sign solutions of the systems (1.1) and (1.2), in $(C[0, T])^{n},\left(C_{l}[0, \infty)\right)^{n}$ and $(B C[0, \infty))^{n}$. Once again, we shall employ an argument originated from Brezis and Browder [11].

Throughout, let $\theta_{i} \in\{-1,1\}, 1 \leq i \leq n$ be fixed. For each $1 \leq j \leq n$, we define

$$
[0, \infty)_{j}=\left\{\begin{array}{l}
{[0, \infty), \quad \theta_{j}=1} \\
(-\infty, 0], \theta_{j}=-1
\end{array}\right.
$$




\subsection{System (1.1)}

Our first result is "parallel" to Theorem 3.2.

Theorem 6.1 Let the following conditions be satisfied for each $1 \leq i \leq n$ : (C1), (C2)(C4) with $p_{i}=\infty$ and $q_{i}=1,(C 5)$, (C6) and (E1)-(E3) where

(E1) $\theta_{i} h_{i}(t) \geq 0$ for $t \in[0, T]$,

(E2) $g_{i}(t, s) \geq 0$ for $s, t \in[0, T]$,

(E3) $\theta_{i} f_{i}(t, u) \geq 0$ for $(t, u) \in[0, T] \times \prod_{j=1}^{n}[0, \infty)_{j}$.

Then, (1.1) has at least one constant-sign solution in $(C[0, T])^{n}$.

Proof First, we shall show that the system

$$
u_{i}(t)=h_{i}(t)+\int_{0}^{T} g_{i}(t, s) f_{i}^{*}(s, u(s)) \mathrm{d} s, \quad t \in[0, T], \quad 1 \leq i \leq n
$$

has a solution in $(C[0, T])^{n}$, where,

$$
f_{i}^{*}\left(t, u_{1}, \ldots, u_{n}\right)=f_{i}\left(t, v_{1}, \ldots, v_{n}\right), \quad t \in[0, T], \quad 1 \leq i \leq n
$$

where for $1 \leq j \leq n$,

$$
v_{j}=\left\{\begin{aligned}
& u_{j}, \theta_{j} u_{j} \geq 0 \\
& 0, \theta_{j} u_{j} \leq 0
\end{aligned}\right.
$$

Clearly, $f_{i}^{*}(t, u):[0, T] \times \mathbb{R}^{n} \rightarrow \mathbb{R}$ and $f_{i}^{*}$ satisfies (C2).

We shall employ Theorem 3.1, so let $u=\left(u_{1}, u_{2}, \ldots, u_{n}\right) \in(C[0, T])^{n}$ be any solution of

$$
u_{i}(t)=\lambda\left(h_{i}(t)+\int_{0}^{T} g_{i}(t, s) f_{i}^{*}(s, u(s)) \mathrm{d} s\right), \quad t \in[0, T], \quad 1 \leq i \leq n
$$

where $\lambda \in(0,1)$. Using (E1)-(E3), we have for $t \in[0, T]$ and $1 \leq i \leq n$,

$$
\theta_{i} u_{i}(t)=\lambda\left(\theta_{i} h_{i}(t)+\int_{0}^{T} g_{i}(t, s) \theta_{i} f_{i}^{*}(s, u(s)) \mathrm{d} s\right) \geq 0 .
$$

Hence, $u$ is a constant-sign solution of $(6.3)_{\lambda}$, and it follows that

$$
f_{i}^{*}(t, u(t))=f_{i}(t, u(t)), \quad t \in[0, T], \quad 1 \leq i \leq n .
$$

Noting (6.4), we see that $(6.3)_{\lambda}$ is the same as $(3.1)_{\lambda}$. Therefore, using a similar technique as in the proof of Theorem 3.2, we obtain (3.15) and subsequently $\|u\| \leq$ $\max _{1 \leq i \leq n} l_{i} \equiv L$. It now follows from Theorem 3.1 (with $M=L+1$ ) that (6.1) has a solution $u^{*} \in(C[0, T])^{n}$.

Noting (E1)-(E3), we have for $t \in[0, T]$ and $1 \leq i \leq n$,

$$
\theta_{i} u_{i}^{*}(t)=\theta_{i} h_{i}(t)+\int_{0}^{T} g_{i}(t, s) \theta_{i} f_{i}^{*}\left(s, u^{*}(s)\right) \mathrm{d} s \geq 0 .
$$

Thus, $u^{*}$ is of constant sign. From (6.2), it is then clear that

$$
f_{i}^{*}\left(t, u^{*}(t)\right)=f_{i}\left(t, u^{*}(t)\right), \quad t \in[0, T], \quad 1 \leq i \leq n .
$$

Hence, $u^{*}$ is actually a solution of (1.1). This completes the proof of the theorem. $\square$ 
Based on the proof of Theorem 6.1, we can develop parallel results to Theorems 3.33.11 as follows.

Theorem 6.2 Let the following conditions be satisfied for each $1 \leq i \leq n$ : (C1), (C2)(C4) with $p_{i}=\infty$ and $q_{i}=1,(C 7),(C 8)$ and (E1)-(E3). Then, (1.1) has at least one constant-sign solution in $(C[0, T])^{n}$.

Theorem 6.3 Let the following conditions be satisfied for each $1 \leq i \leq n$ : (C1), (C2)(C4) with $p_{i}=\infty$ and $q_{i}=1,(C 9)$, (C10) and (E1)-(E3). Then, (1.1) has at least one constant-sign solution in $(C[0, T])^{n}$.

Theorem 6.4 Let the following conditions be satisfied for each $1 \leq i \leq n$ : (C1), (C2)(C4) with $p_{i}=\infty$ and $q_{i}=1,(C 10)-(C 12)$ and (E1)-(E3). Then, (1.1) has at least one constant-sign solution in $(C[0, T])^{n}$.

Theorem 6.5 Let the following conditions be satisfied for each $1 \leq i \leq n$ :(C1)-(C4), (C5), (C10), (C13) and (E1)-(E3). Then, (1.1) has at least one constant-sign solution in $(C[0, T])^{n}$.

Theorem 6.6 Let the following conditions be satisfied for each $1 \leq i \leq n$ :(C1)-(C4), (C7), (C10), (C13) and (E1)-(E3). Then, (1.1) has at least one constant-sign solution in $(C[0, T])^{n}$.

Theorem 6.7 Let the following conditions be satisfied for each $1 \leq i \leq n$ :(C1)-(C4), (C10), (C13), (C14) and (E1)-(E3). Then, (1.1) has at least one constant-sign solution in $(C[0, T])^{n}$.

Theorem 6.8 Let the following conditions be satisfied for each $1 \leq i \leq n$ :(C1)-(C4), (C10), (C13), (C15) and (E1)-(E3). Then, (1.1) has at least one constant-sign solution in $(C[0, T])^{n}$.

Theorem 6.9 Let the following conditions be satisfied for each $1 \leq i \leq n$ :(C1)-(C4), (C10), (C13), (C16) and (E1)-(E3). Then, (1.1) has at least one constant-sign solution in $(C[0, T])^{n}$.

Theorem 6.10 Let the following conditions be satisfied for each $1 \leq i \leq n$ :(C1)-(C4), (C10), (C13), (C17) and (E1)-(E3). Then, (1.1) has at least one constant-sign solution in $(C[0, T])^{n}$.

Remark 6.1 Similar to Remarks 3.1 and 3.2, in Theorem 6.4 the conditions (C10) and (C11) can be replaced by (C10)' and (C11)'; whereas in Theorems 6.5-6.10, (C10) and (C13) can be replaced by (C10)' and (C13)'.

\subsection{System (1.2)}

We shall first obtain the existence of constant-sign solutions of $(1.2)$ in $\left(C_{l}[0, \infty)\right)^{n}$. The first result is "parallel" to Theorem 4.2.

Theorem 6.11 Let the following conditions be satisfied for each $1 \leq i \leq n:$ (D1)-(D5), $(C 5)_{\infty},(C 6)_{\infty}$ and $(E 1)_{\infty}-(E 3)_{\infty}$ where

$(\mathrm{E} 1)_{\infty} \theta_{i} h_{i}(t) \geq 0$ for $t \in[0, \infty)$,

$(\mathrm{E} 2)_{\infty} g_{i}(t, s) \geq 0$ for $s, t \in[0, \infty)$,

(E3) $\theta_{i} f_{i}(t, u) \geq 0$ for $(t, u) \in[0, \infty) \times \prod_{j=1}^{n}[0, \infty)_{j}$.

Then, (1.2) has at least one constant-sign solution in $\left(C_{l}[0, \infty)\right)^{n}$. 
Proof First, we shall show that the system

$$
u_{i}(t)=h_{i}(t)+\int_{0}^{\infty} g_{i}(t, s) f_{i}^{*}(s, u(s)) \mathrm{d} s, \quad t \in[0, \infty), \quad 1 \leq i \leq n
$$

has a solution in $\left(C_{l}[0, \infty)\right)^{n}$. Here,

$$
f_{i}^{*}\left(t, u_{1}, \ldots, u_{n}\right)=f_{i}\left(t, v_{1}, \ldots, v_{n}\right), \quad t \in[0, \infty), \quad 1 \leq i \leq n
$$

where

$$
v_{j}=\left\{\begin{aligned}
u_{j}, \theta_{j} u_{j} & \geq 0 \\
0, \theta_{j} u_{j} & \leq 0
\end{aligned}\right.
$$

Clearly, $f_{i}^{*}(t, u):[0, \infty] \times \mathbb{R}^{n} \rightarrow \mathbb{R}$ and $f_{i}^{*}$ satisfies (D2).

We shall employ Theorem 4.1 , so let $u=\left(u_{1}, u_{2}, \ldots, u_{n}\right) \in\left(C_{l}[0, \infty)\right)^{n}$ be any solution of

$$
u_{i}(t)=\lambda\left(h_{i}(t)+\int_{0}^{\infty} g_{i}(t, s) f_{i}^{*}(s, u(s)) \mathrm{d} s\right), \quad t \in[0, \infty), \quad 1 \leq i \leq n
$$

where $\lambda \in(0,1)$. Then, using a similar technique as in the proof of Theorem 6.1 (and also Theorem 4.2), we can show that (1.2) has a constant-sign solution $u^{*} \in\left(C_{l}\right.$ $[0, \infty))^{n}$. 口

Remark 6.2 Similar to Remark 4.1, in Theorem 6.11 the conditions (D2)-(D5) can be replaced by (D2)'-(D5)'.

Based on the proof of Theorem 6.11, we can develop parallel results to Theorems 4.3-4.5 as follows.

Theorem 6.12 Let the following conditions be satisfied for each $1 \leq i \leq n$ :(D1)-(D5), $(C 7)_{\infty},(C 8)_{\infty}$ and $(E 1)_{\infty}-(E 3)_{\infty}$. Then, (1.2) has at least one constant-sign solution in $\left(C_{l}\right.$ $[0, \infty))^{n}$.

Theorem 6.13 Let the following conditions be satisfied for each $1 \leq i \leq n$ :(D1)-(D5), $(C 9)_{\infty},(C 10)_{\infty}$ and $(E 1)_{\infty}-(E 3)_{\infty}$. Then, (1.2) has at least one constant-sign solution in $\left(C_{l}[0, \infty)\right)^{n}$.

Theorem 6.14 Let the following conditions be satisfied for each $1 \leq i \leq n$ :(D1)-(D5), $(C 10)_{\infty}-(C 12)_{\infty}$ and $(E 1)_{\infty}-(E 3)_{\infty}$. Then, (1.2) has at least one constant-sign solution in $\left(C_{l}[0, \infty)\right)^{n}$.

Remark 6.3 Similar to Remark 4.2, in Theorem 6.14 the conditions $(\mathrm{C} 10)_{\infty}$ and $(\mathrm{C} 11)_{\infty}$ can be replaced by $(\mathrm{C} 10)_{\infty}^{\prime}$ and $(\mathrm{C} 11)_{\infty}^{\prime}$.

We shall now obtain the existence of constant-sign solutions of $(1.2)$ in $(B C[0, \infty))^{n}$. The first result is 'parallel' to Theorem 5.1.

Theorem 6.15 For each $1 \leq i \leq n$, assume (D2)'-(D4)' and (D6). For each $k=1,2, \ldots$, suppose there exists a constant-sign $u^{k}=\left(u_{1}^{k}, u_{2}^{k}, \ldots, u_{n}^{k}\right) \in(C[0, k])^{n}$ that satisfies

$$
u_{i}^{k}(t)=h_{i}(t)+\int_{0}^{k} g_{i}(t, s) f_{i}\left(s, u_{1}^{k}(s), u_{2}^{k}(s), \ldots, u_{n}^{k}(s)\right) \mathrm{d} s, \quad t \in[0, k], \quad 1 \leq i \leq n .
$$


Further, for $1 \leq i \leq n$ and $k=1,2, \ldots$, there is a bounded set $B \subseteq \mathbb{R}$ such that $u_{i}^{k}(t) \in B$ for each $t \in[0, k]$. Then, (1.2) has a constant-sign solution $u^{*} \in(B C[0, \infty))^{n}$ such that for $1 \leq i \leq n, u_{i}^{*}(t) \in \bar{B}$ for all $t \in[0, \infty)$.

Proof Using a similar technique as in the proof of Theorem 5.1, we can show that (5.2) holds. Let $1 \leq i \leq n$. Together with the Arzéla-Ascoli theorem, we obtain subsequences of integers $N_{1}, N_{2}, \ldots$ satisfying (5.3), and functions $z_{i}^{\ell} \in C[0, \ell]$ such that (5.4) holds. Define a function $u_{i}^{*}:[0, \infty) \rightarrow \mathbb{R}$ by (5.5), i.e.,

$$
u_{i}^{*}(t)=z_{i}^{\ell}(t), \quad t \in[0, \ell] .
$$

Since $\theta_{i} u_{i}^{k} \geq 0$, we have $\theta_{i} z_{i}^{\ell} \geq 0$ and so $\theta_{i} u_{i}^{*} \geq 0$. Hence, $u_{i}^{*}$ is of constant sign. The rest of the proof is the same as that of Theorem 5.1.

The next result is "parallel" to Theorem 5.2.

Theorem 6.16 Let (D2)-(D4) and (D6) be satisfied for each $1 \leq i \leq n$. Moreover, suppose the following conditions hold for each $1 \leq i \leq n$ and each $w \in\{1,2, \ldots\}:,(C 5)_{w}$, $(C 6)_{w}$ and $(E 1)_{w}-(E 3)_{w}$ where

$(\mathrm{E} 1)_{w} \theta_{i} h_{i}(t) \geq 0$ for $t \in[0, w]$,

$(\mathrm{E} 2)_{w} g_{i}(t, s) \geq 0$ for $s, t \in[0, w]$,

$(\mathrm{E} 3)_{w} \theta_{i} f_{i}(t, u) \geq 0$ for $(t, u) \in[0, w] \times \prod_{j=1}^{n}[0, \infty)_{j}$.

Then, (1.2) has at least one constant-sign solution in $(B C[0, \infty))^{n}$.

Proof We shall apply Theorem 6.15. To do so, for $w=1,2, \ldots$, we shall show that the system (5.8) has a constant-sign solution $u^{*}$ in $(C[0, w])^{n}$. The proof of this is similar to that of Theorem 6.1 (with $T=w$ ) and Theorem 5.2. As in (5.9) we have $\left|u_{i}^{*}(t)\right| \leq l_{i}$ for each $t \in[0, w]$ and $1 \leq i \leq n$. All the conditions of Theorem 6.15 are now satisfied and the conclusion is immediate. $\square$

Based on the proof of Theorem 6.16, we can develop parallel results to Theorems 5.3-5.5 as follows:

Theorem 6.17 Let (D2)-(D4) and (D6) be satisfied for each $1 \leq i \leq n$. Moreover, suppose the following conditions hold for each $1 \leq i \leq n$ and each $w \in\{1,2, \ldots\}:(C 7)_{w}$ $(C 8)_{w}$ and $(E 1)_{w}-(E 3)_{w}$. Then, $(1.2)$ has at least one constant-sign solution in $(B C[0, \infty))$ $n$.

Theorem 6.18 Let (D2)-(D4) and (D6) be satisfied for each $1 \leq i \leq n$. Moreover, suppose the following conditions hold for each $1 \leq i \leq n$ and each $w \in\{1,2, \ldots\}:(C 9)_{w}$, $(C 10)_{w}$ and $(E 1)_{w}-(E 3)_{w}$. Then, (1.2) has at least one constant-sign solution in $(B C[0$, $\infty))^{n}$.

Theorem 6.19 Let (D2)-(D4) and (D6) be satisfied for each $1 \leq i \leq n$. Moreover, suppose the following conditions hold for each $1 \leq i \leq n$ and each $w \in\{1,2, \ldots\}:(C 11)_{w}$, $(C 12)_{w}$ and $(E 1)_{w}-(E 3) w$. Then, (1.2) has at least one constant-sign solution in $(B C[0$, $\infty))^{n}$.

Remark 6.4 Similar to Remark 5.1, in Theorem 6.19 the conditions $(\mathrm{C} 10)_{w}$ and (C11) ${ }_{w}$ can be replaced by $(\mathrm{C} 10)_{w}^{\prime}$ and $(\mathrm{C} 11)_{w}^{\prime}$.

\section{Examples}

We shall now illustrate the results obtained through some examples. 
Example 7.1 In system (1.1), consider the following $f_{i}, 1 \leq i \leq n$ :

$$
f_{i}(t, u)= \begin{cases}\kappa_{i}(t, u), & u \in P \\ 0, & \text { otherwise. }\end{cases}
$$

Here,

$$
P=\left\{u \in(C[0, T])^{n}: u_{1}(t), u_{2}(t), \ldots, u_{n}(t)>c \text { for all } t \in[0, T]\right\}
$$

where $c>0$ is a given constant, and $\kappa_{i}$ is such that

(a) the map $u \alpha f_{i}(t, u)$ is continuous for almost all $t \in[0, T]$;

(b) the map $t \alpha f_{i}(t, u)$ is measurable for all $u \in \mathbb{R}^{n}$;

(c) for any $r>0$, there exists $\mu_{r, i} \in L^{1}[0, T]$ such that $|u| \leq r$ implies $\left|\kappa_{i}(t, u)\right| \leq \mu_{r, i}$

(t) for almost all $t \in[0, T]$;

(d) for any $u \in P, u_{\mathrm{i}}(t) \kappa_{i}(t, u(t)) \geq 0$ for all $t \in[0, T]$.

Next, suppose for each $1 \leq i \leq n$,

$$
h_{i} \in C[0, T] \text { with } H_{i} \equiv \sup _{t \in[0, T]}\left|h_{i}(t)\right|<c .
$$

Clearly, conditions (C1) and (C2) with $q_{i}=1$ are fulfilled. We shall check that condition (C6) is satisfied. Pick $r>c$ and $\alpha_{i}=\frac{c}{r}, 1 \leq i \leq n$. Then, from (7.2) we have $r \alpha_{i}=c$ $>H_{i}$.

Let $u \in P$. Then, from (7.1) we have $f_{i}(t, u)=\kappa_{i}(t, u)$. Consider $\|u(t)\|>r$ where $t \in$ $[0, T]$. If $\| u(t)||=\left|u_{i}(t)\right|$, then noting (d) we have

$$
\begin{aligned}
u_{i}(t) f_{i}(t, u(t)) & =\left|u_{i}(t)\right| \cdot\left|f_{i}(t, u(t))\right|=|| u(t)|| \cdot\left|f_{i}(t, u(t))\right| \\
& >r\left|f_{i}(t, u(t))\right| \\
& >r \cdot \frac{c}{r} \cdot\left|f_{i}(t, u(t))\right| \\
& =r \alpha_{i}\left|f_{i}(t, u(t))\right| .
\end{aligned}
$$

If $\|u(t)\|=\left|u_{k}(t)\right|$ for some $k \neq i$, then

$$
\begin{aligned}
u_{i}(t) f_{i}(t, u(t)) & =\left|u_{i}(t)\right| \cdot\left|f_{i}(t, u(t))\right|=r \cdot \frac{\left|u_{i}(t)\right|}{r} \cdot\left|f_{i}(t, u(t))\right| \\
& >r \cdot \frac{c}{r} \cdot\left|f_{i}(t, u(t))\right| \\
& =r \alpha_{i}\left|f_{i}(t, u(t))\right| .
\end{aligned}
$$

Therefore, from (7.3) and (7.4) we see that condition (C6) holds for $u \in P$.

For $u \in(C[0, T])^{n} \backslash P$, we have $f_{i}(t, u)=0$ and (C6) is trivially true. Hence, we have shown that condition (C6) is satisfied.

The next example considers a convolution kernel $g_{i}(t, s)$ which arises in nonlinear diffusion and percolation problems; the particular case when $n=1$ has been investigated by Bushell and Okrasiński [26].

Example 7.2 Consider system (1.1) with (7.1), (7.2), and for $1 \leq i \leq n$,

$$
g_{i}(t, s)=(t-s)^{\gamma_{i}-1}
$$

where $\gamma_{i}>1$. 
Clearly, $g_{i}$ satisfies (C3) and (C4) with $p_{i}=\infty$. Next, we shall check condition (C5). For $u \in P(P$ is given in Example 7.1), we have

$$
\begin{aligned}
\int_{0}^{T}\left[f_{i}(t, u(t)) \int_{0}^{T} g_{i}(t, s) f_{i}(s, u(s)) \mathrm{d} s\right] \mathrm{d} t & =\int_{0}^{T}\left[\kappa_{i}(t, u(t)) \int_{0}^{T}(t-s)^{\gamma_{i}-1} \kappa_{i}(s, u(s)) \mathrm{d} s\right] \mathrm{d} t \\
& \leq T^{\gamma_{i}-1} \int_{0}^{T}\left[\kappa_{i}(t, u(t)) \int_{0}^{T} \kappa_{i}(s, u(s)) \mathrm{d} s\right] \mathrm{d} t \\
& \leq B_{i}
\end{aligned}
$$

since $\kappa_{i}(t, u)$ satisfies (c) (note (c) is stated in Example 7.1). This shows that condition (C5) holds for $u \in P$. For $u \in(C[0, T])^{n} \backslash P$, we have $f_{i}(t, u)=0$ and (C5) is trivially true. Therefore, condition (C5) is satisfied.

It now follows from Theorem 3.2 that the system (1.1) with (7.1), (7.2) and (7.5) has at least one solution in $(C[0, T])^{n}$.

The next example considers an $g_{i}(t, s)$ of which the particular case when $n=1$ originates from the well known Emden differential equation.

Example 7.3 Consider system (1.1) with (7.1), (7.2), and for $1 \leq i \leq n$,

$$
g_{i}(t, s)=(t-s) s^{\gamma_{i}}
$$

where $\gamma_{i} \geq 0$.

Clearly, $g_{i}$ satisfies (C3) and (C4) with $p_{i}=\infty$. Next, we see that condition (C5) is satisfied. In fact, for $u \in P$, corresponding to (7.6) we have

$$
\begin{aligned}
\int_{0}^{T}\left[f_{i}(t, u(t)) \int_{0}^{T} g_{i}(t, s) f_{i}(s, u(s)) \mathrm{d} s\right] \mathrm{d} t & =\int_{0}^{T}\left[\kappa_{i}(t, u(t)) \int_{0}^{T}(t-s) s^{\gamma_{i}} \kappa_{i}(s, u(s)) \mathrm{d} s\right] \mathrm{d} t \\
& \leq T^{\gamma_{i}+1} \int_{0}^{T}\left[\kappa_{i}(t, u(t)) \int_{0}^{T} \kappa_{i}(s, u(s)) \mathrm{d} s\right] \mathrm{d} t \\
& \leq B_{i} .
\end{aligned}
$$

Hence, by Theorem 3.2 the system (1.1) with (7.1), (7.2) and (7.7) has at least one solution in $(C[0, T])^{n}$.

Our next example illustrates the existence of a positive solution in $(C[0, T])^{n}$, this is the particular case of constant-sign solution usually considered in the literature.

Example 7.4 Let $\theta_{i}=1,1 \leq i \leq n$. Consider system (1.1) with (7.1), (7.2), and for $1 \leq$ $i \leq n$,

$$
h_{i}(t) \geq 0, \quad t \in[0, T] .
$$

Clearly, condition (E1) is met, and noting (d) in Example 7.1 condition (E3) is also fulfilled. Moreover, both $g_{i}(t, s)$ in (7.5) and (7.7) satisfy condition (E2). From Examples 7.1-7.3, we see that all the conditions of Theorem 6.1 are met. Hence, we conclude that

the system (1.1) with (7.1), (7.2), (7.9) and (7.5).

and 
the system (1.1) with (7.1), (7.2), (7.9) and (7.7).

each of which has at least one positive solution in $(C[0, T])^{n}$.

Example 7.5 In system (1.2), consider the following $f_{i}, 1 \leq i \leq n$ :

$$
f_{i}(t, u)= \begin{cases}\kappa_{i}(t, u), & u \in P_{\infty} \\ 0, & \text { otherwise }\end{cases}
$$

Here,

$$
P_{\infty}=\left\{u \in\left(C_{l}[0, \infty)\right)^{n}: u_{1}(t), u_{2}(t), \ldots, u_{n}(t)>c \text { for all } t \in[0, \infty)\right\}
$$

where $c>0$ is a given constant, and $\kappa_{i}$ is such that

(a) $)_{\infty}$ the map $u \alpha f_{i}(t, u)$ is continuous for almost all $t \in[0, \infty)$;

(b) $)_{\infty}$ the map $t \alpha f_{i}(t, u)$ is measurable for all $u \in \mathbb{R}^{n}$;

(c) $)_{\infty}$ for any $r>0$, there exists $\mu_{r, i} \in L^{1}[0, \infty)$ such that $|u| \leq r$ implies $\left|\kappa_{i}(t, u)\right| \leq$ $\mu_{r, i}(t)$ for almost all $t \in[0, \infty)$;

$(\mathrm{d})_{\infty}$ for any $u \in P_{\infty}, u_{i}(t) \kappa_{i}(t, u(t)) \geq 0$ for all $t \in[0, \infty)$.

Next, suppose for each $1 \leq i \leq n$,

$$
h_{i} \in C_{l}[0, \infty) \text { with } H_{i} \equiv \sup _{t \in[0, \infty)}\left|h_{i}(t)\right|<c .
$$

Clearly, conditions (D1) and (D2) are satisfied. Moreover, using a similar technique as in Example 7.1, we see that condition (C6) $)_{\infty}$ is satisfied.

Example 7.6 Consider system (1.2) with (7.10), (7.11), and for $1 \leq i \leq n$,

$$
g_{i}(t, s)=\frac{1}{s+1}+\frac{1}{(1+t)^{\gamma_{i}}}
$$

where $\gamma_{i} \geq 1$.

Clearly, $g_{i}$ satisfies (D3), (D4) and (D5) (take $\left.\tilde{g}_{i}(s)=\frac{1}{s+1}\right)$. Next, we shall check condition $(\mathrm{C} 5)_{\infty}$. For $u \in P_{\infty}\left(P_{\infty}\right.$ is given in Example 7.5), we have

$$
\begin{aligned}
& \int_{0}^{\infty}\left[f_{i}(t, u(t)) \int_{0}^{\infty} g_{i}(t, s) f_{i}(s, u(s)) \mathrm{d} s\right] \mathrm{d} t \\
& \quad=\int_{0}^{\infty}\left[\kappa_{i}(t, u(t)) \int_{0}^{\infty}\left(\frac{1}{s+1}+\frac{1}{(1+t)^{\gamma i}}\right) \kappa_{i}(s, u(s)) \mathrm{d} s\right] \mathrm{d} t \\
& \quad \leq 2 \int_{0}^{\infty}\left[\kappa_{i}(t, u(t)) \int_{0}^{\infty} \kappa_{i}(s, u(s)) \mathrm{d} s\right] \mathrm{d} t \leq B_{i}
\end{aligned}
$$

since $\kappa_{i}(t, u)$ satisfies $(\mathrm{c})_{\infty}$ (note $(\mathrm{c})_{\infty}$ is stated in Example 7.5). This shows that condition (C5) $)_{\infty}$ holds for $u \in P_{\infty}$. For $u \in\left(C_{l}[0, \infty)\right)^{n} \backslash P_{\infty}$, we have $f_{i}(t, u)=0$ and (C5) is trivially true. Hence, condition $(C 5)_{\infty}$ is satisfied.

We can now conclude from Theorem 4.2 that the system (1.2) with (7.10), (7.11) and (7.12) has at least one solution in $\left(C_{l}[0, \infty)\right)^{n}$. 
The next example shows the existence of a positive solution in $\left(C_{l}[0, \infty)\right)^{n}$, this is the special case of constant-sign solution usually considered in the literature.

Example 7.7 Let $\theta_{i}=1,1 \leq i \leq n$. Consider system (1.2) with (7.10)-(7.12), and for 1 $\leq i \leq n$,

$$
h_{i}(t) \geq 0, \quad t \in[0, \infty) .
$$

Clearly, conditions $(E 1)_{\infty}-(E 3)_{\infty}$ are satisfied. Noting Examples 7.5 and 7.6, we see that all the conditions of Theorem 6.11 are met. Hence, the system (1.2) with (7.11)(7.12) has at least one positive solution in $\left(C_{l}[0, \infty)\right)^{n}$.

\section{Acknowledgements}

The authors would like to thank the referee for the comments which help to improve the paper.

\section{Author details}

${ }^{1}$ Department of Mathematics, Texas A\&M University - Kingsville, Kingsville, TX 78363, USA ${ }^{2}$ Department of Mathematics, Faculty of Science, King Abdulaziz University, 21589 Jeddah, Saudi Arabia ${ }^{3}$ Department of Mathematics, National University of Ireland, Galway, Ireland ${ }^{4}$ School of Electrical and Electronic Engineering, Nanyang Technological University 50 Nanyang Avenue, Singapore 639798, Singapore

\section{Authors' contributions}

All authors contributed equally to the manuscript and read and approved the final draft.

\section{Competing interests}

The authors declare that they have no competing interests.

Received: 18 March 2011 Accepted: 11 October 2011 Published: 11 October 2011

\section{References}

1. Erbe, LH, Hu, S, Wang, H: Multiple positive solutions of some boundary value problems. J Math Anal Appl. 184, 640-648 (1994). doi:10.1006/jmaa.1994.1227

2. Erbe, $\mathrm{LH}$, Wang, H: On the existence of positive solutions of ordinary differential equations. Proc Am Math Soc. 120, 743-748 (1994). doi:10.1090/50002-9939-1994-1204373-9

3. Lian, W, Wong, F, Yeh, C: On the existence of positive solutions of nonlinear second order differential equations. Proc Am Math Soc. 124, 1117-1126 (1996). doi:10.1090/S0002-9939-96-03403-X

4. Agarwal, RP, Meehan, M, O'Regan, D: Nonlinear Integral Equations and Inclusions. Nova Science Publishers, Huntington, NY (2001)

5. O'Regan, D, Meehan, M: Existence Theory for Nonlinear Integral and Integrodifferential Equations. Kluwer, Dordrecht (1998)

6. Agarwal, RP, O'Regan, D, Wong, PJY: Constant-sign solutions of a system of Fredholm integral equations. Acta Appl Math. 80, 57-94 (2004)

7. Agarwal, RP, O'Regan, D, Wong, PJY: Eigenvalues of a system of Fredholm integral equations. Math Comput Modell. 39 1113-1150 (2004). doi:10.1016/50895-7177(04)90536-5

8. Agarwal, RP, O'Regan, D, Wong, PJY: Triple solutions of constant sign for a system of Fredholm integral equations. Cubo. 6, 1-45 (2004)

9. Agarwal, RP, O'Regan, D, Wong, PJY: Constant-sign solutions of a system of integral equations: The semipositone and singular case. Asymptotic Anal. 43, 47-74 (2005)

10. Agarwal, RP, O'Regan, D, Wong, PJY: Constant-sign solutions of a system of integral equations with integrable singularities. J Integral Equ Appl. 19, 117-142 (2007). doi:10.1216/jiea/1182525211

11. Brezis, H, Browder, FE: Existence theorems for nonlinear integral equations of Hammerstein type. Bull Am Math Soc. 81, 73-78 (1975). doi:10.1090/50002-9904-1975-13641-X

12. Agarwal, RP, O'Regan, D, Wong, PJY: Positive Solutions of Differential, Difference and Integral Equations. Kluwer Academic Publishers, Dordrecht (1999)

13. Anselone, PM, Lee, JW: Nonlinear integral equations on the half line. J Integral Equ Appl. 4, 1-14 (1992). doi:10.1216/ jiea/1181075663

14. Anselone, PM, Sloan, IH: Integral equations on the half line. J Integral Equ. 9, 3-23 (1985)

15. Bushell, PJ: On a class of Volterra and Fredholm non-linear integral equations. Math Proc Cambridge Philos Soc. 79 , 329-335 (1976). doi:10.1017/\$0305004100052324

16. Corduneanu, C: Integral Equations and Applications. Cambridge University Press, New York (1990)

17. Krasnosel'skii, MA: Topological Methods in the Theory of Nonlinear Integral Equations. Pergamon Press, Oxford (1964)

18. Meehan, M, O'Regan, D: Existence theory for nonlinear Fredholm and Volterra integral equations on half-open intervals. Nonlinear Anal. 35, 355-387 (1999). doi:10.1016/50362-546X(97)00719-0

19. Nashed, MZ, Wong, JSW: Some variants of a fixed point theorem of Krasnosel'skii and applications to nonlinear integral equations. J Math Mech. 18, 767-777 (1969)

20. O'Regan, D: Existence results for nonlinear integral equations. J Math Anal Appl. 192, 705-726 (1995). doi:10.1006/ jmaa.1995.1199 
21. O'Regan, D: Existence results for nonlinear integral equations on the half line. In: Corduneanu C (ed.) Qualitative problems for differential equations and control theory. pp. 121-131. World Scientific Publishing, River Edge, NJ (1995)

22. O'Regan, D: A fixed point theorem for condensing operators and applications to Hammerstein integral equations in Banach spaces. Comput Math Appl. 30L, 39-49 (1995)

23. O'Regan, D: Volterra and Urysohn integral equations in Banach spaces. J Appl Math Stochastic Anal. 11, 449-464 (1998). doi:10.1155/S1048953398000379

24. Dugundji, J, Granas, A: Fixed Point Theory. Monografie Mathematyczne, PWN Warsaw (1982)

25. Lee, JW, O'Regan, D: Existence principles for nonlinear integral equations on semi-infinite and half-open intervals. In: Sivasundarem S, Martynyuk AA (eds.) Advances in Nonlinear Dynamics. pp. 355-364. Gordon and Breach Science Publishers, Amsterdam (1997)

26. Bushell, PJ, Okrasiński, W: Uniqueness of solutions for a class of nonlinear Volterra integral equations with convolution kernel. Math Proc Cambridge Philos Soc. 106, 547-552 (1989). doi:10.1017/S0305004100068262

doi:10.1186/1687-1847-2011-43

Cite this article as: Agarwal et al:: Existence results of Brezis-Browder type for systems of Fredholm integral equations. Advances in Difference Equations 2011 2011:43.

\section{Submit your manuscript to a SpringerOpen ${ }^{\circ}$} journal and benefit from:

- Convenient online submission

- Rigorous peer review

- Immediate publication on acceptance

- Open access: articles freely available online

- High visibility within the field

- Retaining the copyright to your article

Submit your next manuscript at $\gg$ springeropen.com 